\title{
Effects of velocity shear layer on detonation propagation in a supersonic expanding combustor
}

DOI:

$10.1063 / 5.0065348$

Document Version

Accepted author manuscript

Link to publication record in Manchester Research Explorer

\section{Citation for published version (APA):}

Dai, J., Xu, F., Cai, X., \& Mahmoudi Larimi, Y. (2021). Effects of velocity shear layer on detonation propagation in a supersonic expanding combustor. Physics of Fluids. https://doi.org/10.1063/5.0065348

\section{Published in:}

Physics of Fluids

\section{Citing this paper}

Please note that where the full-text provided on Manchester Research Explorer is the Author Accepted Manuscript or Proof version this may differ from the final Published version. If citing, it is advised that you check and use the publisher's definitive version.

\section{General rights}

Copyright and moral rights for the publications made accessible in the Research Explorer are retained by the authors and/or other copyright owners and it is a condition of accessing publications that users recognise and abide by the legal requirements associated with these rights.

\section{Takedown policy}

If you believe that this document breaches copyright please refer to the University of Manchester's Takedown Procedures [http://man.ac.uk/04Y6Bo] or contact uml.scholarlycommunications@manchester.ac.uk providing relevant details, so we can investigate your claim.

\section{OPEN ACCESS}




\title{
Effects of velocity shear layer on detonation propagation in a supersonic expanding combustor
}

\author{
Jian Dai, ${ }^{1}$ Fei Xu, ${ }^{1}$ Xiaodong Cai, ${ }^{2}$ a and Yasser Mahmoudi ${ }^{3}$ \\ ${ }^{1}$ School of Aeronautics and Astronautics, Central South University, Changsha 410083, \\ China \\ ${ }^{2}$ Science and Technology on Scramjet Laboratory, National University of Defense \\ Technology, Changsha 410073, China \\ ${ }^{3}$ Department of Mechanical, Aerospace and Civil Engineering, The University of \\ Manchester, M13 9PL, United Kingdom \\ ${ }^{a}$ Corresponding author: cai-chonger@hotmail.com
}

Abstract: This study investigates the mechanism of detonation propagation in a stoichiometric hydrogen-oxygen mixture with non-uniform flow velocity entering an expanding combustor. For simulation of the detonation propagation, the NavierStokes equations with a one-step two-species chemistry model are solved by employing the hybrid sixth-order weighted essentially non-oscillatory centre difference scheme. The self-sustaining mechanism of detonation propagation in an expanding combustor under the action of non-uniform supersonic flow with a velocity shear layer is revealed. The results show that under the influence of velocity shear layer, two different unburned jets are produced behind the detonation front. These jets are induced by the velocity shear layers and the Prandtl-Meyer expansion fan. The two jets interact and mix gradually. The interaction between the mixed unburned jets and highly unstable shear layers creates large-scale vortices that intensify the 
turbulent mixing of the unburned jets. Meanwhile, the baroclinic mechanism generates numerous vortices on the boundary of the unburned jet. These vortices promote the mixing of the burned and unburned gases, which eventually leads to the rapid consumption of the unburned pockets. The heat released due to the burning of the unreacted pockets behind the detonation wave, supports a self-sustaining propagation of the detonation wave. When the velocity difference among the shear layers increases, the surface fluctuation of the detonation wave increases.

Keywords: Supersonic expanding channel; Self-sustaining detonation propagation; Velocity shear layer; Unburned jet

\section{Introduction}

The scramjet is an important part of future hypersonic air-breathing propulsion systems because of its excellent thrust performance. ${ }^{1}$ However, the existing scramjet technologies are based on the isobaric combustion cycle, which hinders the significant improvement in the engine thrust performance. ${ }^{2,3}$ In recent years, a major breakthrough in the near-isochoric combustion cycle of the detonation engine has been achieved. Theoretically, the thrust performance of the detonation engine exceeds that of the scramjet by more than $30 \%,{ }^{4,5}$ highlighting the prime significant development in the future hypersonic propulsion systems using detonation engine. ${ }^{6,7}$

Among previous studies, a series of experimental and numerical investigations have been conducted on detonation combustion in supersonic flow. These have primarily focused on the detonation initiation and its propagation in fully straight channels, ${ }^{8,9}$ straight channels with cavity ${ }^{10}$ and obstacle ${ }^{11}$, and expanding channels ${ }^{12}$. 
In reality, to obtain higher thrust performance and improve the outlet velocity, the supersonic thrust chamber is usually configured with an expanding channel. ${ }^{13,14}$ Subbotin ${ }^{15}$ experimentally observed that the detonation transition can be triggered by the interaction between the deflagration flame front and expansion wave generated at the entrance of the expanding channel. The expansion wave, which has a highpressure gradient, accelerates combustion process by promoting the mixing of unburned and burned gases. Tunik et al. ${ }^{16,17}$ investigated the detonation combustion of kerosene vapours in an expanding nozzle. They ${ }^{16,17}$ observed that with an increase in the Mach number of the incoming flow, the combustion efficiency of kerosene detonation decreases due to the formation of a flow separation zone near the expansion wall. Wang et al. ${ }^{18}$ reported that the interaction between the oblique detonation wave and expansion fan induces a new wave structure in the expanding channel. Cai et al. ${ }^{12,19}$ conducted a numerical simulation of detonation in an expanding channel. They ${ }^{12,19}$ demonstrated that the heat release from the combustion of the unburned jet behind the detonation front, promotes dynamic and stable propagation of the detonation wave. The rapid jet consumption resulted in appearance of fluctuations and pressure oscillations in the flow field, eventually attenuating the detonation wave.

Most of the above studies considered detonation structures in ideal homogeneous combustible mixtures. In a real hypersonic flight condition, the flow properties into the combustor is usually variable and non-uniform. ${ }^{20-22}$ This occurs because of various factors, including non-uniform mixing, imperfect fuel evaporation, ${ }^{23}$ pressure 
fluctuations at the inlet, ${ }^{24}$ and the influence of boundary layers on combustor walls. ${ }^{25,26}$ To date, numerous valuable conclusions have been derived from the study of detonation with non-uniform combustible mixtures in a straight channel. The uneven distribution of flow parameters has been found to considerably influence the detonation wave propagation. ${ }^{27-29}$ Song et al. ${ }^{30}$ numerically studied self-sustaining modes of detonations in inhomogeneous mixtures of hydrogen and oxygen. They ${ }^{30}$ found that there are different self-sustaining detonation modes at low, medium, and high concentration gradients. Kuznetsov et al. $^{31}$ indicated that the detonation behaviours influenced by the concentration gradient mainly depends on the gradient sharpness. Relatively sharp gradients consistently result in the detonation attenuation and even its failure. By contrast, detonation can achieve self-sustaining propagation influenced by a relatively smooth gradient. ${ }^{31}$ Kim et al. ${ }^{32}$ demonstrated that increasing the temperature gradient in non-uniform temperature regions reduces the temperature of the unburned mixture, thereby decreasing the combustion wave speed. Cai et al..$^{33,34}$ reported that the inhomogeneity of velocity and the chemical reactivity of the mixture can significantly affect the formation and development of detonation waves.

In this work, the aim is to gain deep insight into the turbulent characteristics of the flow behind the detonation wave in a combustor with the inclusion of the incoming nonuniform flow velocity. The existing studies in the literature were dedicate primarily to the systems where the inflow properties to the combustor is uniform, which does not replicate the condition occur in realistic detonation engines. Especially, when an aircraft accelerates or decelerates, the flow properties entering the engine combustor changes, 
leading to an uneven distribution of the flow velocity field. ${ }^{35,36}$ The shear layers arising from velocity difference will act on detonation wave and affect the rapid combustion of the mixture behind such wave. ${ }^{34}$ Hence, in this work for the first time, we aim to explore the role of fine turbulent flow features such as hydrodynamic instability (RichtmyerMeshkov) and their interaction with the unstable shear layers and unburned jets in expanding channels with non-uniform incoming flow, which will directly or indirectly affect the structure and propagation mode of detonation wave. By monitoring the development of unburned jet behind detonation wave, the internal relationship between its generation and consumption mechanism and self-sustaining propagation of blast wave is explored. Additionally, the present work for the first time aims at analysing the detonations stabilization in a supersonic channel by controlling the inlet flow conditions compared to the CJ condition, and further, gain deep insight to the consumption mechanism of unburned gases through turbulent mixing initiated by the Richtmeyer Meshkov instability. An in-depth understanding of these mechanisms can help to effectively control the detonation wave in supersonic combustor, which is vital for developing efficient combustion organization technology. Hence, it is indeed of great guiding significance for the practical application of detonation engines to explore fundamentally the mechanisms of detonation propagation, stabilisation in expanding combustors with non-uniform supersonic flow. In the present work, the mechanism of self-sustaining detonation propagation with layered velocity in expanding channels is investigated using the dynamic adaptive mesh refinement program AMROC (Adaptive Mesh Refinement in Object-Oriented C++), ${ }^{37-40}$ which has been 
demonstrated in multiple dimensional detonation simulations ${ }^{41,42}$.

\section{Numerical method}

In this section, the details of the numerical method, including computational model, mesh generation, and governing equations deployed to analyse the detonation propagation in an expanding channel are presented.

\subsection{Model configuration}

The computational model of the expanding combustor is shown in Fig. 1. A stoichiometric mixture of hydrogen-oxygen $\left(\mathrm{H}_{2}-\mathrm{O}_{2}\right)$ with a molar ratio of $2: 1$ is selected as the supersonic inflow to the combustor model and the properties summarised in Table 1. The supersonic flow in Fig. 1 travels from right to left at the corresponding Chapman-Jouguet (CJ) velocity. Hence, the right side of the domain is prescribed inflow condition with the parameters of the supersonic combustible mixture. The ideal outflow condition (implemented by extrapolating ghost cells from the internal flow) is specified on the left boundary. ${ }^{19}$ The upper and lower walls adopt reflecting boundaries with slip adiabatic wall conditions. The level-set technique with the ghost fluid method is employed on the upper expanding wall. The hot jet injection method $^{43}$ is used for detonation initiation as shown in Fig. 1. The parameters of the hot jet are associated with the $\mathrm{H}_{2}-\mathrm{O}_{2}$ detonation properties in the $\mathrm{CJ}$ equilibrium state (see Table 2). After a successful detonation initiation, the hot jet inflow condition immediately transforms into the reflecting boundary condition. 


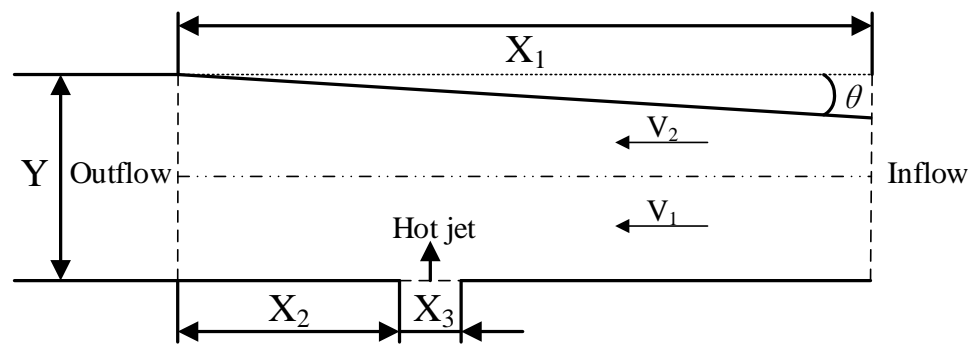

Fig. 1 Calculation model $\left(X_{1}=75 \mathrm{~mm} ; X_{2}=25 \mathrm{~mm} ; X_{3}=4 \mathrm{~mm} ; Y=25 \mathrm{~mm} ; \theta=3^{\circ}\right)$.

Table 1 Basic inflow parameters.

\begin{tabular}{lll}
\hline Parameters & Values & Unit \\
\hline$P_{\infty}$ & 6.67 & $\mathrm{kPa}$ \\
$T_{\infty}$ & 300 & $\mathrm{~K}$ \\
$\rho_{\infty}$ & 0.077552 & $\mathrm{~kg} / \mathrm{m}^{3}$ \\
$\gamma$ & 1.29499 & - \\
$V_{\infty}$ & 1587.84 (CJ detonation velocity) & $\mathrm{m} / \mathrm{s}$ \\
$W$ (Molecular quantity) & 0.029 & $\mathrm{~kg} / \mathrm{mol}$ \\
$q$ (Heat release per unit mass) & 54000 & $\mathrm{~J} / \mathrm{mol}$ \\
$E_{a}$ (Activation energy) & 30000 & $\mathrm{~J} / \mathrm{mol}$ \\
$A$ (Frequency factor) & $6 \times 10^{5}$ & $\mathrm{~s}-1$ \\
\hline
\end{tabular}

Table 2 Parameters of the hot jet in the equilibrium CJ state (at $P_{\infty}=6.67 \mathrm{kPa}$ and $T_{\infty}=300 \mathrm{~K}$ ).

\begin{tabular}{lll}
\hline Parameters & Values & Unit \\
\hline$P_{C J}$ & 86376 & $\mathrm{~Pa}$ \\
$T_{C J}$ & 1943.8 & $\mathrm{~K}$ \\
$\rho_{C J}$ & 0.155 & $\mathrm{~kg} / \mathrm{m}^{3}$ \\
$V_{j}$ & 850 (speed of sound) & $\mathrm{m} / \mathrm{s}$ \\
$e$ (Total energy per unit mass) & 349280 & $\mathrm{~J} / \mathrm{mol}$ \\
$Y_{1}$ (Mass fraction of reactant) & 0.0088 & - \\
$Y_{2}$ (Mass fraction of product) & 0.9912 & - \\
\hline
\end{tabular}

After a stable detonation is formed in the channel, the supersonic flow in Fig. 1 is divided into two different velocity layers at the inlet of the model to emphasize the effects of significant velocity differences on the detonation propagation. The mixture in the lower half part of the channel flows at velocity $V_{1}$ and that in the upper half 
flows at velocity $V_{2}$ with various cases listed in Table 3. Case 1 is the basic example of the clarifying mechanism of the self-sustaining detonation propagation. Due to the asymmetry of the channel used in the analysis, the distribution of the velocity shear layers needs to be considered. The other cases are set with $\mathrm{V}_{1}<\mathrm{V}_{2}, \mathrm{~V}_{1}>\mathrm{V}_{2}$, and $\mathrm{V}<$ $V_{C J}, V=V_{C J}, V>V_{C J}$, where $V$ is the average velocity of inlet, i.e., $V=\left(V_{1}+V_{2}\right) / 2$.

Table 3 Parameter setting of velocity shear layers.

\begin{tabular}{ccccccccc}
\hline \multirow{2}{*}{ Case } & 1 & 2 & 3 & 4 & 5 & 6 & 7 & 8 \\
\cline { 2 - 8 } & \multicolumn{2}{c}{$\mathrm{V}<\mathrm{V}_{\mathrm{CJ}}$} & \multicolumn{2}{c}{$\mathrm{V}=\mathrm{V}_{\mathrm{CJ}}$} & \multicolumn{3}{c}{$\mathrm{V}>\mathrm{V}_{\mathrm{CJ}}$} \\
\hline $\mathrm{V}_{2} / \mathrm{V}_{\mathrm{CJ}}$ & 0.9 & 1.0 & 0.9 & 1.1 & 1.0 & 1.1 & 1.1 & 1.15 \\
$\mathrm{~V}_{1} / \mathrm{V}_{\mathrm{CJ}}$ & 1.0 & 0.9 & 1.1 & 0.9 & 1.1 & 1.0 & 1.15 & 1.1 \\
\hline
\end{tabular}

\subsection{Governing equations and numerical scheme}

In the expanding channel, unburned jets are usually generated behind the detonation wave. The consumption of an unburned jet is closely related to turbulent mixing and diffusion, which directly affect the self-sustaining propagation of detonation. Hence, to account for these physics, the Navier-Stokes (NS) equations are adopted as the governing equations as follows:

$$
\frac{\partial \boldsymbol{U}}{\partial t}+\frac{\partial \boldsymbol{F}_{c o n v}}{\partial x}+\frac{\partial \boldsymbol{H}_{c o n v}}{\partial y}=\frac{\partial \boldsymbol{F}_{\text {diff }}}{\partial x}+\frac{\partial \boldsymbol{H}_{\text {diff }}}{\partial y}+\boldsymbol{S}_{\text {chem }},
$$

where the state vector $\boldsymbol{U}$, the convective flux vectors $\boldsymbol{F}_{\text {conv }}$ and $\boldsymbol{H}_{c o n v}$, the diffusive flux vectors $\boldsymbol{F}_{\text {diff }}$ and $\boldsymbol{H}_{\text {diff }}$, and the reactive source vector are, respectively given as

$$
\begin{gathered}
\boldsymbol{U}=\left[\begin{array}{c}
\rho \\
\rho u \\
\rho v \\
\rho e \\
\rho Y_{1}
\end{array}\right], \boldsymbol{F}_{c o n v}=\left[\begin{array}{c}
\rho u \\
\rho u^{2}+p \\
\rho u v \\
(\rho e+p) u \\
\rho u Y_{1}
\end{array}\right], \boldsymbol{H}_{c o n v}=\left[\begin{array}{c}
\rho v \\
\rho u v \\
\rho v^{2}+p \\
(\rho e+p) v \\
\rho v Y_{1}
\end{array}\right], \\
\boldsymbol{F}_{d i f f}=\left[\begin{array}{c}
\tau_{x x} \\
\tau_{x y} \\
u \tau_{x x}+v \tau_{x y}+k \frac{\partial \boldsymbol{T}}{\partial x}+\rho h_{1} D_{1} \frac{\partial \boldsymbol{Y}_{1}}{\partial x}+\rho h_{2} D_{2} \frac{\partial \boldsymbol{Y}_{2}}{\partial x} \\
\rho D_{1} \frac{\partial \boldsymbol{Y}_{\mathbf{1}}}{\partial x}
\end{array}\right],
\end{gathered}
$$




$$
\boldsymbol{H}_{\text {diff }}=\left[\begin{array}{c}
0 \\
\tau_{y x} \\
\tau_{y y} \\
u \tau_{y x}+v \tau_{y y}+k \frac{\partial \boldsymbol{T}}{\partial y}+\rho h_{1} D_{1} \frac{\partial \boldsymbol{Y}_{1}}{\partial y}+\rho h_{2} D_{2} \frac{\partial \boldsymbol{Y}_{2}}{\partial y} \\
\rho D_{1} \frac{\partial \boldsymbol{Y}_{1}}{\partial y} \\
\boldsymbol{S}_{\text {chem }}=\left(0,0,0,0, \dot{\omega}_{1}\right)^{T} .
\end{array}\right]
$$

In the above equations, $\rho$ is the total density. $u$ and $v$ are the velocity in the $\mathrm{x}$ direction and y-direction, respectively. $p$ and $T$ are the pressure and temperature, respectively. Furthermore, $h_{1}$ and $h_{2}$ are the enthalpies of the reactant and product, respectively. $k, D_{1}$ and $D_{2}$ are the thermal conductivity, the mass diffusivities of the reactant and the product, respectively. $\dot{\omega}_{1}$ is the mass production rate of the reactant, and $e$ is the total energy per unit mass, defined as

$$
e=\frac{p}{\rho(\gamma-1)}+\frac{\left(u^{2}+v^{2}\right)}{2}+Y_{1} q
$$

$\tau$ is the stress tensor, whose components are

$$
\tau_{x x}=\mu\left(\frac{4}{3} \frac{\partial u}{\partial x}-\frac{2}{3} \frac{\partial v}{\partial y}\right), \tau_{x y}=\tau_{y x}=\mu\left(\frac{\partial v}{\partial x}+\frac{\partial u}{\partial y}\right) \text { and } \tau_{y y}=\mu\left(\frac{4}{3} \frac{\partial v}{\partial y}-\frac{2}{3} \frac{\partial u}{\partial x}\right),
$$

where $\mu$ is the mixture viscosity coefficient.

Considering simplified chemical kinetics, the one-step two-species reaction model $^{44}$ was utilized in the simulation. For the two species, the calorically perfect model is

$$
\gamma=\gamma_{1}=\gamma_{2}, p=\rho R T, R=R_{1}=R_{2} .
$$

The mass fraction production rates are given as follows:

$$
\dot{\omega}_{1}=-\dot{\omega}_{2}=\rho Y_{1} A \exp \left(\frac{E_{a}}{R T}\right)
$$

At the end of the Zel'dovich-von Neumann-Döring (ZND) reaction zone, the temperature and pressure are about $2500 \mathrm{~K}$ and $101.325 \mathrm{kPa}$, respectively. The transport parameters for the one-step model are given by: $T_{\text {ref }}=2500 \mathrm{~K}, \mu_{\text {ref }}=$ $1.07 \times 10^{-4} \mathrm{~Pa} \cdot \mathrm{s}, k_{\mathrm{ref}}=0.148 \mathrm{~W} /(\mathrm{m} \cdot \mathrm{K}), D_{1 \mathrm{ref}}=5.5 \times 10^{-4} \mathrm{~m}^{2} / \mathrm{s}, \mathrm{D}_{2 \mathrm{ref}}=6.4 \times 10^{-4}$ 
$\mathrm{m}^{2} / \mathrm{s}$. The viscosity $(\mu)$ and conductivity $(k)$ are given by the Sutherland model. The mass diffusion coefficient $(D)$ is given by a simplified formula inversely proportional to pressure as:

$$
\begin{gathered}
\frac{\mu}{\mu_{\text {ref }}}=\left(\frac{T}{T_{\text {ref }}}\right)^{5 / 2}, \frac{k}{k_{\text {ref }}}=\left(\frac{T}{T_{\text {ref }}}\right)^{5 / 2}, \\
\frac{D_{1}}{D_{1 \text { ref }}}=\frac{p_{\text {atm }}}{p}\left(\frac{T}{T_{\text {ref }}}\right)^{5 / 2}, \frac{D_{2}}{D_{\text {2ref }}}=\frac{p_{\text {atm }}}{p}\left(\frac{T}{T_{\text {ref }}}\right)^{5 / 2} .
\end{gathered}
$$

Although there are certain differences in the detonation structures between twodimensional and three-dimensional simulations,${ }^{45}$ the two-dimensional simulation can also well represent the initiation and propagation process of the detonation wave. ${ }^{46}$

The typical methods for shock-capturing introduce considerable numerical dissipation, which disturbs the physical diffusion part of the NS equations. To analyse the complex structure of flow field with the inclusion of various shock waves more accurately, the hybrid sixth-order weighted essentially non-oscillatory centered difference (WENO-CD) scheme $\mathrm{s}^{47,48}$ is utilised to solve the NS equations with chemical reaction. The hybrid WENO-CD scheme consists of two parts: the finite difference sixth-order WENO scheme, which is applied to the shock discontinuity, and the conservative central difference sixth-order CD scheme, which is applied to the smooth region. ${ }^{49}$ This numerical scheme can well represent the robustness of WENO at the shock discontinuity. Moreover, it can maintain the CD characteristics without numerical dissipation in the smooth region and thus reduce the numerical dissipation to the extent feasible.

\subsection{Mesh refinement verification}

To evaluate the independence of the grids, it is necessary to compare the 
obtained results with different mesh refinement levels. The initial mesh size adopted in this study is $600 \times 200$, with the initial mesh refinement of $1.25 \times 10^{-4} \mathrm{~m}$. Adaptive mesh technology improves the mesh accuracy by refining the mesh level by level. Each colour in Fig. 2 represents a refinement level. Under the present flow conditions, the half reaction length of the one-dimensional ZND model is $\mathrm{Lhr}_{\mathrm{h}}=2.145 \mathrm{~mm}$. With three-level, four-level and five-level refinements, grid resolutions of 68.64, 137.28, and 274.56 points per half reaction length $(\mathrm{Pts} / \mathrm{Lhr})$, respectively, were achieved. The specific mesh refinement parameters are shown in Table 4. It was previously demonstrated that the resolution of $40 \mathrm{Pts} / \mathrm{Lhr}_{\mathrm{hr}}$ is needed to clearly resolved the twodimensional detonation simulations. ${ }^{45}$ According to the above analyses, the mesh resolution of these three mesh refinement types is higher than $40 \mathrm{Pts} / \mathrm{Lhr}$. In Fig. 2, the field characteristics, including bow shock, Mach reflection, shock-induced combustion, transverse wave, and the secondary reflected shock, are clearly resolved properly using the three-level, four-level, and five-level refinements. For more detailed comparison, the pressure profile of the flow field was intercepted at $\mathrm{Y}=20$ mm. Fig. 3 shows the pressure distribution along this line in three flow fields. We can directly see that the local detonation wave position of 4-level refinement is basically consistent with that of 5-level refinement. However, in contrast, the local detonation wave position under the 3-level refinement has obvious deviation, which is about 0.6 mm earlier than that under the other two levels. It is then concluded that both level 4 and level 5 refinement measures can reasonably achieve reliable results. To balance the calculation time and grid accuracy, the second highest resolution, four-level 
refinement, is selected for the following calculations.
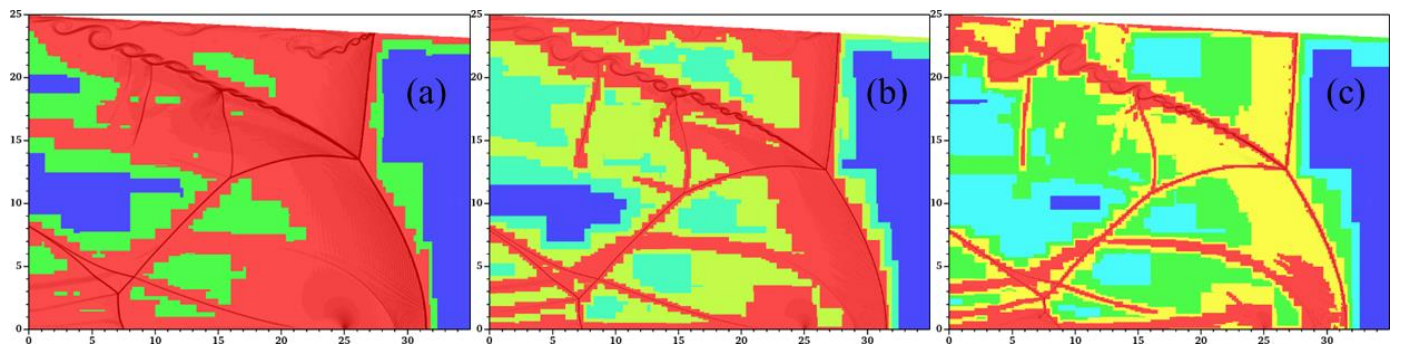

Fig. 2 Three types of adaptive mesh refinement distribution. (a) Three-level refinement, (b) four-level refinement, (c) five-level refinement.

Table 4 Mesh refinement parameters.

\begin{tabular}{llll}
\hline & Three-level & Four-level & Five-level \\
\hline Refinement factors & $\mathrm{r}_{1}=2, \mathrm{r}_{2}=2$ & $\mathrm{r}_{1}=2, \mathrm{r}_{2}=2, \mathrm{r}_{3}=2$ & $\mathrm{r}_{1}=2, \mathrm{r}_{2}=2, \mathrm{r}_{3}=2, \mathrm{r}_{4}=2$ \\
Nodes & 749519 & 1862986 & 4105175 \\
Zones & 705743 & 1764661 & 3896287 \\
$\Delta_{\min }$ & $3.12 \times 10^{-5} \mathrm{~m}$ & $1.56 \times 10^{-5} \mathrm{~m}$ & $7.8 \times 10^{-6} \mathrm{~m}$ \\
Resolution & $68.64 \mathrm{Pts} / \mathrm{L}_{\mathrm{hr}}$ & $137.28 \mathrm{Pts} / \mathrm{L}_{\mathrm{hr}}$ & $274.56 \mathrm{Pts} / \mathrm{L}_{\mathrm{hr}}$ \\
\hline
\end{tabular}

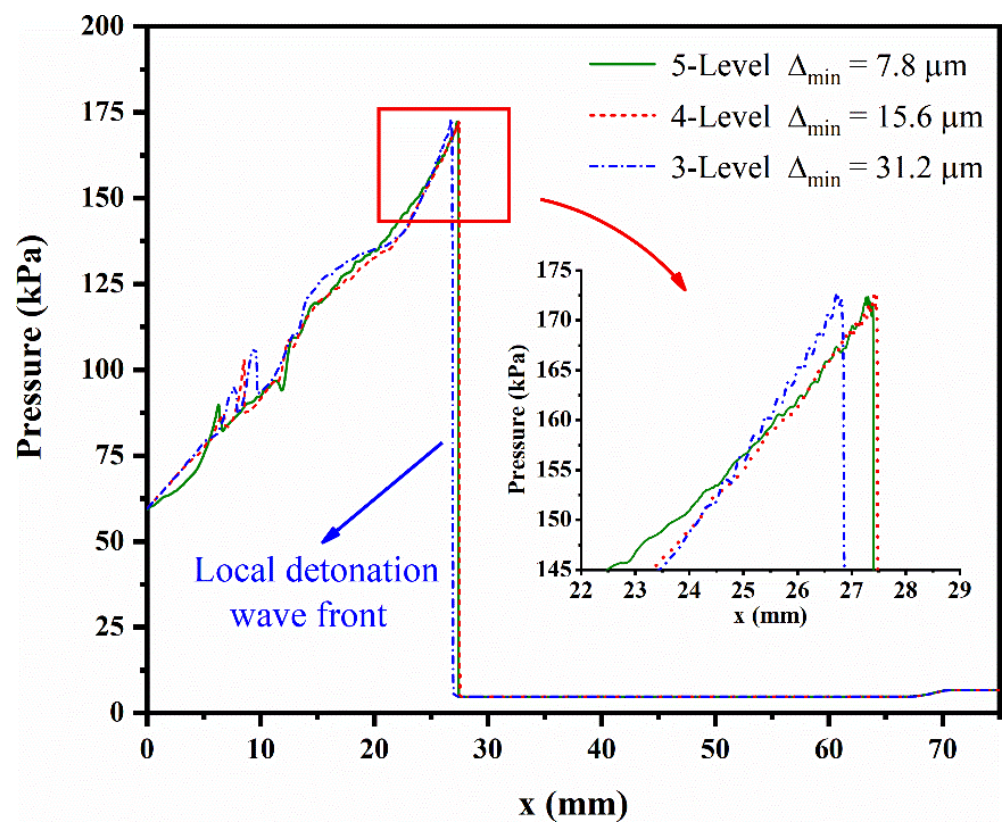

Fig. 3 Flow field pressure profiles with three grid scales

Fig. 4 shows the effects of adaptive mesh refinement on detonation initiation 
process. The top row shows the pressure contours, and the bottom row shows the refinement effects of four-level. As illustrated in Fig. 4, after the hot jet is injected into the supersonic combustible mixture, a bow shock wave is induced rapidly. Soon the bow shock is reflected on the upper wall and gradually forms a Mach reflection, which is actually a local detonation wave. As the triple-point moves downward along the bow shock, the detonation combustion of the whole channel forms eventually. It is seen in Fig. 4 that with the development and change of the flow, the program adaptively refines and thins the corresponding grids, efficiently realizing the adaptive refining calculation of the dynamic flow field. Previously, Chen et al. ${ }^{12}$ performed some experimental analysis to analyze detonation front features in an expanding channel deploying hot jet detonation initiation. Comparing the numerical schlieren obtained in the present work against their experimental data ${ }^{12}$, the overall structure of the numerical result is qualitatively similar to the experiment result, especially in resolving the bow shock, transverse wave, and Mach stem. 

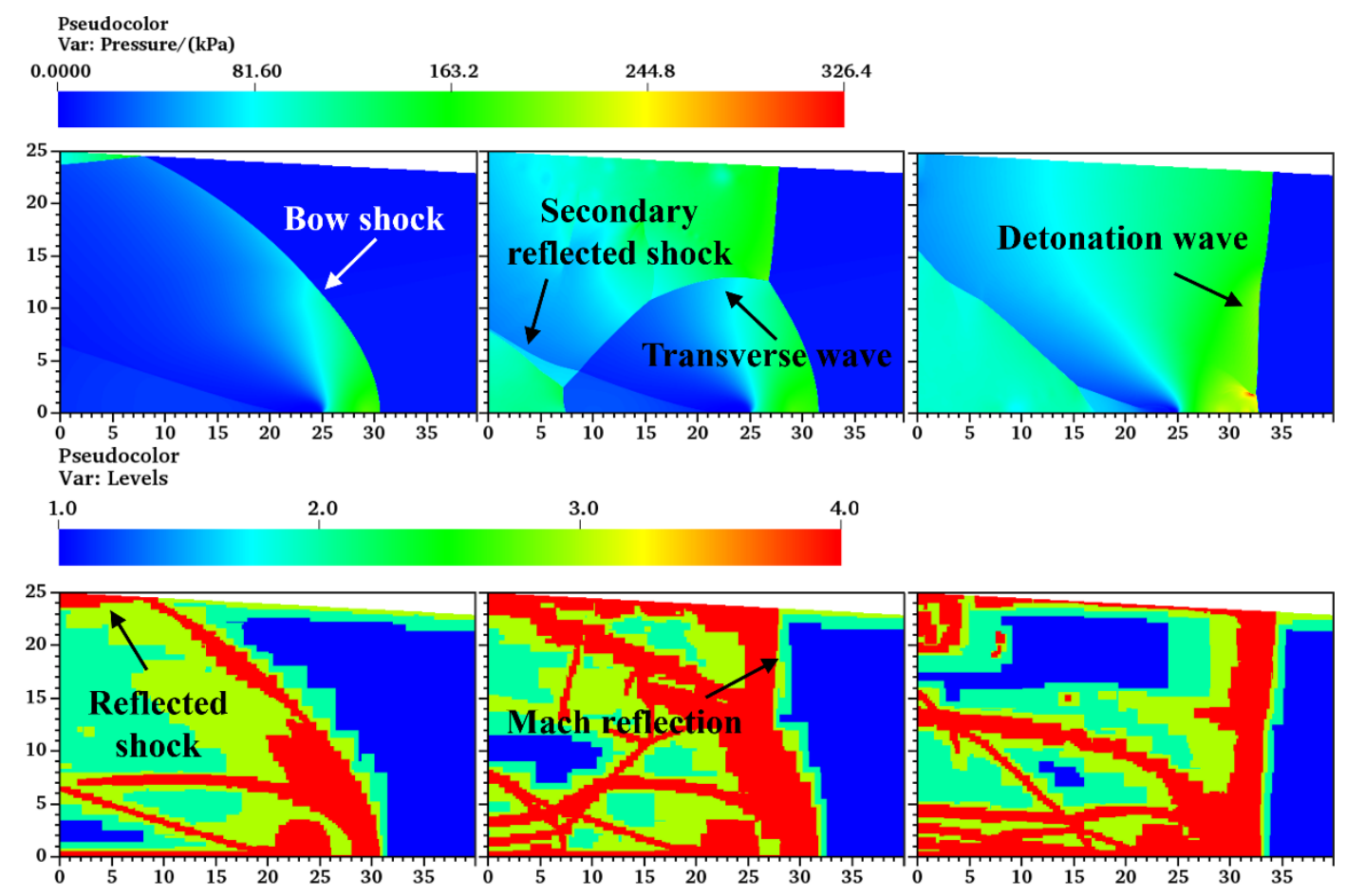
(a) $\mathrm{t}=135 \mu \mathrm{s}$
(b) $\mathrm{t}=225 \mu \mathrm{s}$
(c) $\mathbf{t}=260 \mu \mathrm{s}$

Fig. 4 Pressure contours (top row) and corresponding refinement level distributions (bottom row)

showing adaptive mesh refinement effects in detonation initiation process, (a) $t=135 \mu \mathrm{s}$, (b) $t=225 \mu \mathrm{s}$,

$$
\text { (c) } \mathrm{t}=260 \mu \mathrm{s} \text {. }
$$

\section{Results and discussions}

In the following sections, Case 1 in Table 3 is considered as the default example in section 3.1 and 3.2, demonstrating the analysis of the detonation phenomenon and the mechanism in the expanding combustor with a layered inlet velocity. The example of uniform inlet velocity (i.e., $\mathrm{V}_{1}=\mathrm{V}_{2}=\mathrm{V}_{\mathrm{CJ}}$ ) in Ref. [19] is used to assist the analyses.

\subsection{Propagation process of detonation wave}

The contours of product mass fractions of the detonation propagation for Case 1 in Table 3 are shown in Fig. 5. As illustrated in Fig. 5(a), because of the influence of the Prandtl-Meyer expansion fan $^{50}$ and velocity shear layer, two different unburned 
jets are generated behind the detonation wave, and the stability of the detonation wave is weakened. This characteristic does not exist if the velocity is uniform ${ }^{19}$. Fig. 5(a) shows two triple points on the detonation front with the upper triple point moving upward along the shock front towards the vicinity of the unburned jets. Concurrently, the lower triple point reflects on the straight wall. It is seen in Fig. 5(a) that a typical 'mushroom cloud' vortex structure is generated by the interaction of the separated shear layers behind the triple points, because of the Richtmyer-Meshkov (RM) instability. As the detonation wave propagates forward, the two unburned jets gradually mix and extend downstream simultaneously. They are continuously lifted up to the expanding wall by the alternating actions of the shear layers. Near the expanding wall, a separated shock wave is observed in Fig. 5(b). Subsequently, when the last triple point reaches the upper wall, a part of the unburned jets forms unburned pockets near the expanding wall. The remaining unburned jets gradually separate from the triple point and extend downstream in Fig. 5(c). It is seen that the shear layers interact with the unburned jets and induce numerous large-scale vortices, resulting in the appearance of turbulence feature in the flow field, which occurs at local Reynolds number higher than $10^{4} .{ }^{51}$ The local Reynolds number $\left(R e=\frac{\rho v \delta}{\mu}\right)$ of the flow is estimated using the average density $(\rho)$, velocity $(v)$, and the local transverse extent of the turbulent region $(\delta)$ at the top and bottom of the unsteady shear layer on the interface of the unburned jet. Dimotakis ${ }^{51}$ indicated that the transition Reynolds $R e_{t r}=1-2 \times 10^{4}$ is a sign of development of turbulence. The Reynolds numbers in Fig. 5(a), (b), (c), and (d) are $9.0 \times 10^{4}, 1.7 \times 10^{5}, 8.6 \times 10^{4}$, and 
$2.4 \times 10^{4}$, respectively. Among them, the Reynolds number in Fig. 5(b) is an order of magnitude larger than the transition value. It is demonstrated that the interface of unburned jet is turbulent with the action of unstable shear layer, which promotes its mixing and consumption. As shown in Fig. 5(d), new unburned jets are generated with the continuous consumption of residual unburned pockets. Moreover, the propagation of the whole detonation wave presents an asynchronous dynamic mode, i.e., first, the detonation front in the upper half of the flow field tilts forward, then the lower half catches up with that in the upper half of the flow field, and the upper half tilts forward again, form a cycle. The highly unstable shear layer along the unburned jet can be considered as a free boundary, ${ }^{52}$ and the motion of this free boundary indirectly affects the propagation of the detonation wave.

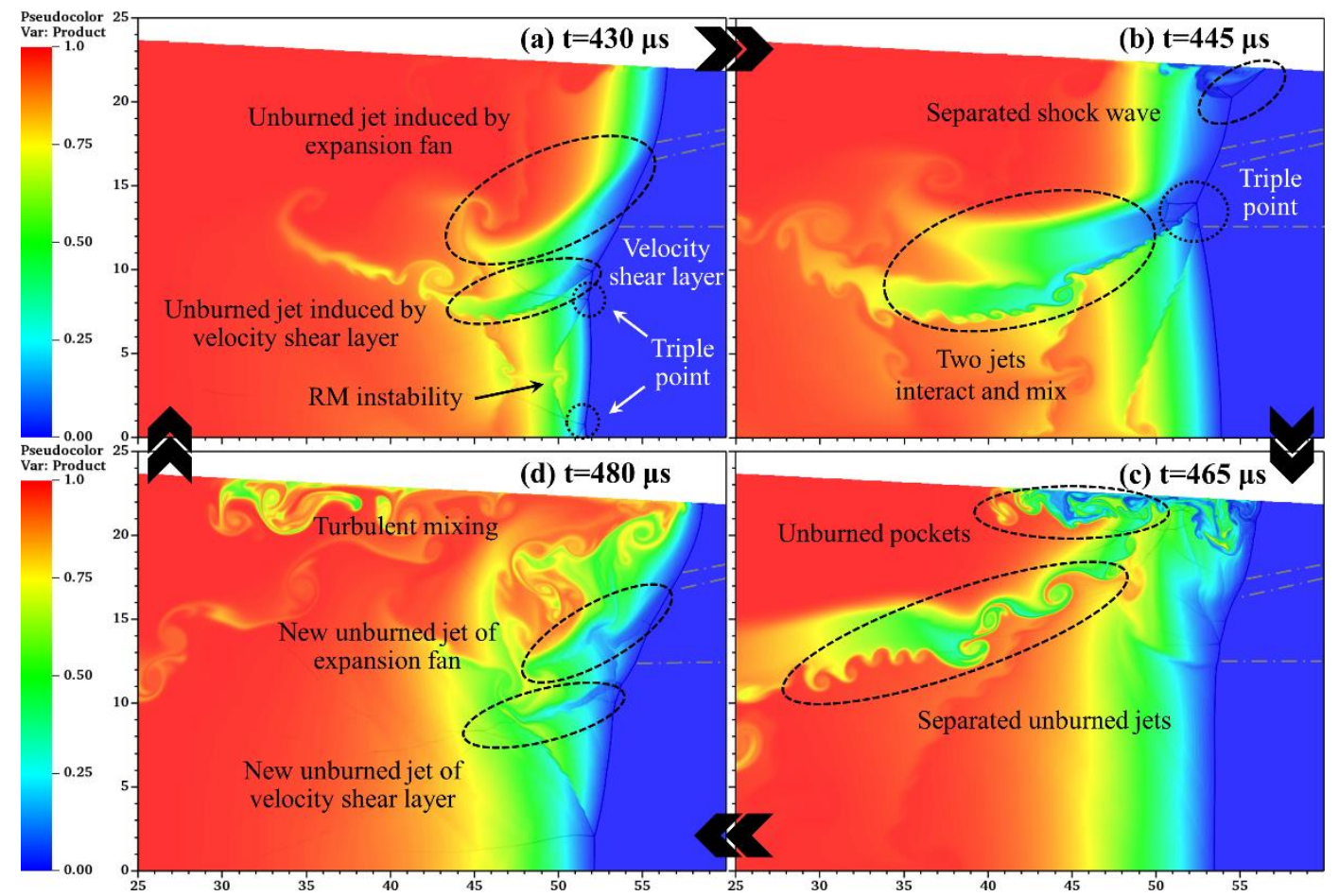

Fig. 5 Contours of product mass fractions showing detonation propagation in expanding channel with velocity shear layer for Case 1 in Table 3 , (a) $t=430 \mu \mathrm{s}$, (b) $t=445 \mu \mathrm{s}$, (c) $t=465 \mu \mathrm{s}$, (d) $\mathrm{t}=480 \mu \mathrm{s}$. 
As depicted in Fig. 6, the trajectory of the detonation front in the case of layered velocity is compared with that of the case with uniform inlet velocity. The trajectory of the detonation front is determined by capturing the $\mathrm{x}$-coordinate of the maximum pressure gradient of $\mathrm{y}=12.5 \mathrm{~mm}$ in each frame. The detonation front under the uniform flow condition gradually attenuates, whereas that under the layered flow condition, the detonation wave continues to propagate forward. The foregoing occurs because the total pressure in the upper half of the flow field is modified by the introduction of the velocity shear layer. At $\mathrm{t}=380 \mu \mathrm{s}$, the velocity of the upper half of the flow field suddenly decreases, resulting in the reduction of dynamic pressure; however, the static pressure remains constant. Thus, the relative Mach number of the detonation wave increases and the detonation wave propagates forward. The detonation front trajectory of the layered velocity approximates a straight line. This demonstrates the formation of dynamic self-sustaining detonation in the expanding channel with a fitting slope of 0.03035 corresponding to a relative velocity of 30.35 $\mathrm{m} / \mathrm{s}$. At the same time, the detonation wave for layered velocity is accompanied by a periodic oscillation and the period is estimated to be $T_{\text {oscillation period }}=65 \mu \mathrm{s}$. It is speculated that the periodic oscillation of the front is closely related to the periodic generation and consumption of unburned jets behind the detonation wave. 


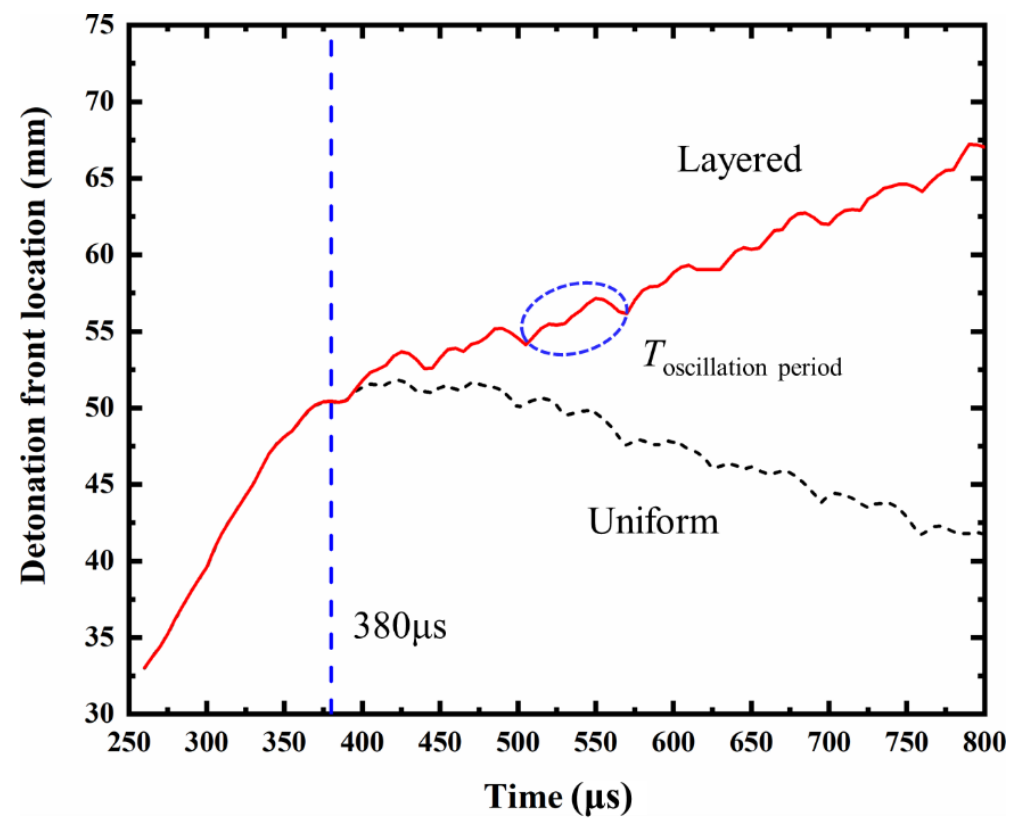

Fig. 6 Detonation front trajectories with layered velocity of Case 1 and the uniform example in Ref.

[19].

\subsection{Mechanism of self-sustaining propagation}

The Rankine-Hugoniot equation given by Eq. $(12)^{53}$ reveals the generation of unburned jets:

$$
p=p_{\infty} \times\left(\frac{2 \gamma_{\infty}}{\gamma_{\infty}+1} \mathrm{M} a_{\infty}^{2} \sin ^{2} \beta-\frac{\gamma_{\infty}-1}{\gamma_{\infty}+1}\right)
$$

Note that for an oblique or bow shock wave, the shock strength is related to three parameters: incoming supersonic pressure $\left(p_{\infty}\right)$, Mach number $\left(\mathrm{M} a_{\infty}\right)$, and tangent angle $(\beta)$ of the local shock. Due to effect of the expansion channel, the flow direction through the Prandtl-Meyer expansion fan is deflected thereby decreasing the tangent angle of the local shock wave and incoming pressure..$^{50}$ This directly weakens the strength of the detonation wave. Moreover, its adiabatic compression leads to an incomplete consumption of the combustible mixture, resulting in the formation of an unburned jet behind the detonation front. ${ }^{54}$ Similarly, after the velocity stratification, 
the velocity of the upper half of the flow field is reduced to a value less than the initial velocity, resulting in reduced detonation strength, thus forming unburned jets.

Fig. 7 shows the density distributions of the flow fields with uniform and layered inlet velocities. To prove the weakening effects of the expansion fan and velocity shear layer on the detonation wave, the density peaks at the upper, middle and lower parts of the channel are compared in Fig. 7. It is seen in Fig. 7(c) that the peak density along line " $\mathrm{A}-\mathrm{A}$ " is $0.345 \mathrm{~kg} / \mathrm{m}^{3}$, which is smaller than the peak density along line "C-C" $\left(0.468 \mathrm{~kg} / \mathrm{m}^{3}\right)$. The peak density decreases by $26.28 \%$ due to the expansion fan. This indicates that compared with the position " $\mathrm{A}-\mathrm{A}$ ", along the position " $\mathrm{C}-\mathrm{C}$ ", the reactant is compressed more strongly by the shock front, demonstrating the expansion fan weakens the strength of detonation wave. Without the influence of the expansion fan and velocity shear layer, the peak density along line " $\mathrm{C}-\mathrm{C}$ " is equal to that along line " $\mathrm{F}-\mathrm{F}$ ". The density peak value of $0.444 \mathrm{~kg} / \mathrm{m}^{3}$ along " $\mathrm{B}-\mathrm{B}$ " is $11.84 \%$ higher than the density peak value of $0.397 \mathrm{~kg} / \mathrm{m}^{3}$ along "E-E", meaning that the decrease of velocity in the upper half of the flow field also weakens the strength of detonation wave. However, affected by both the expansion fan and the velocity shear layer, the peak density along the position " $\mathrm{D}-\mathrm{D}$ " is $0.337 \mathrm{~kg} / \mathrm{m}^{3}$, which is $27.99 \%$ lower than that along the position " $\mathrm{F}-\mathrm{F}$ ". Hence, the generation of the unburned jets are closely related to the effects of the expansion fan and the velocity shear layer. 


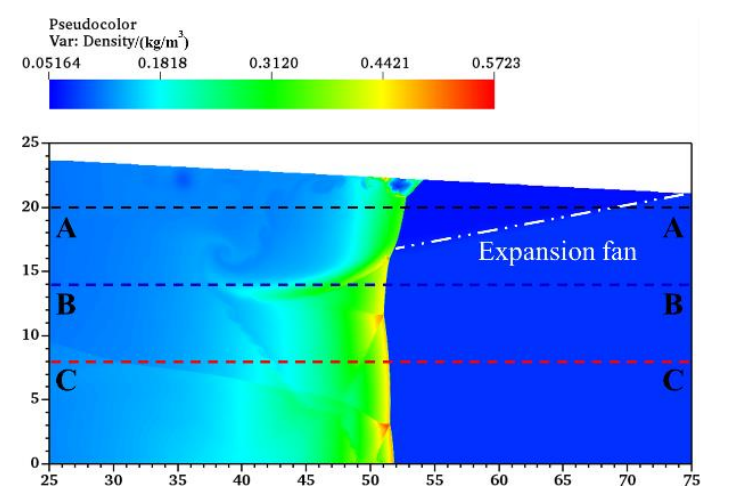

(a)

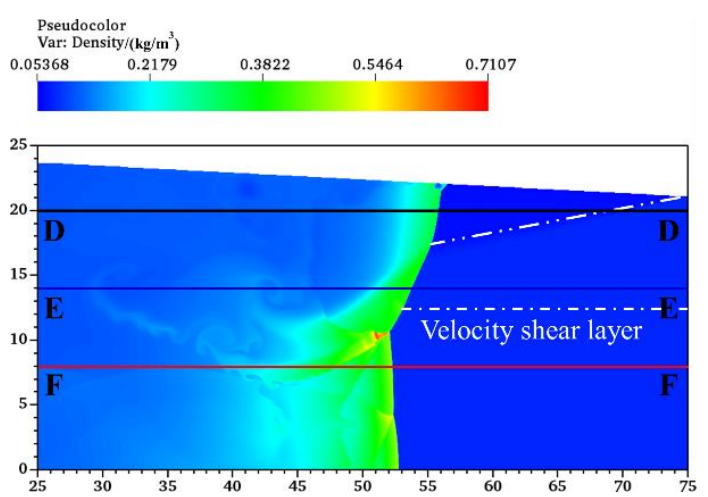

(b)

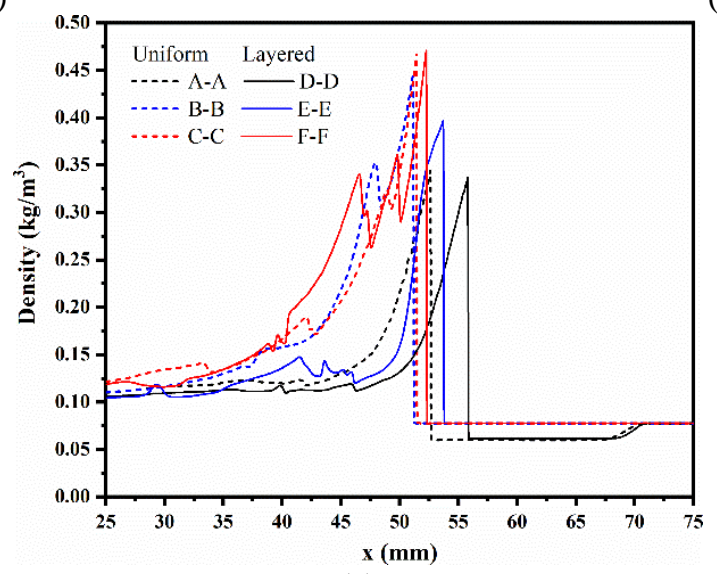

(c)

Fig. 7 Density distributions of the flow fields at $t=435 \mu \mathrm{s}$, (a) the density contour of the uniform example, (b) the density contour of Case 1, (c) Density curves at six positions shown in (a) and (b).

The velocity vectors of the flow field and contours of the product mass fractions are superimposed in Fig. 8(a), showing the relationship between the flow direction and the free boundary of the unburned jets in the flow field. The velocity vectors shown in Fig. 8(a) indicate that the flow field behind the detonation front flows downward while passing through the free boundary of the unburned jet. This suggests that this free boundary compresses the space in the lower half of the flow field behind the detonation front, forming a micro-compression channel, in which the expansion of the detonation product is suppressed. This increases the pressure behind the detonation front in the lower half of the flow field, pushing forward the detonation 
wave in the lower half of the domain from line (1) to line (2) and (3) (see Fig. 8(b)). Finally, the detonation wave in the lower half of the flow field catches up with that in the upper half of the flow field, again completely forming a whole detonation wave (line (4)), as seen in Fig. 8(b).
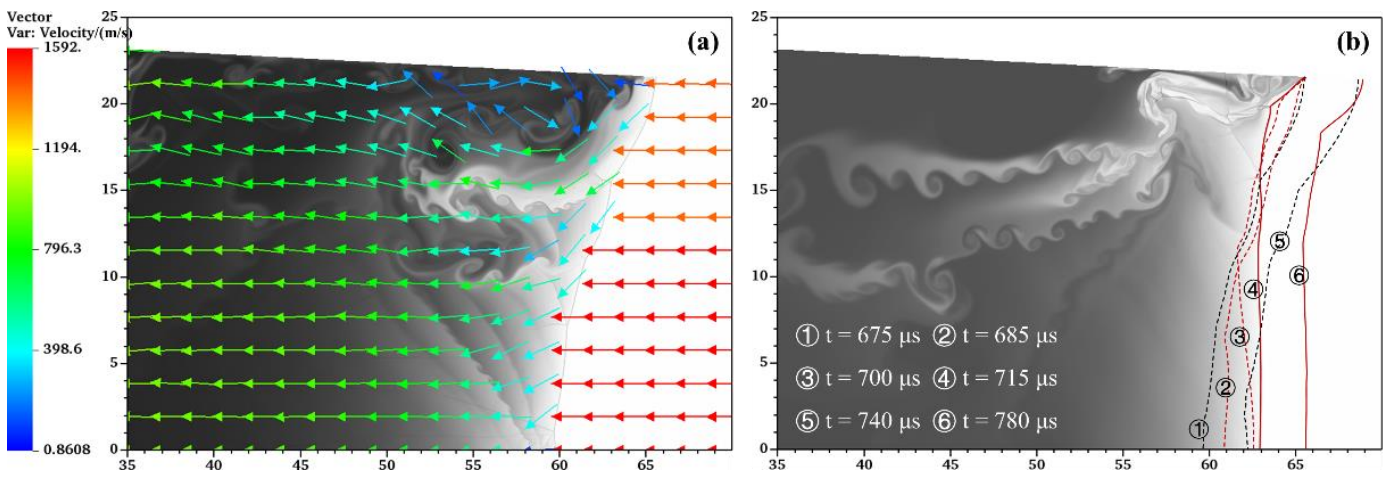

Fig. 8 The motion of detonation front of Case 1, (a) contours of product mass fractions and velocity vectors at $\mathrm{t}=675 \mu \mathrm{s}$, (b) dynamic trajectories of detonation propagation.

To further demonstrate the mechanism of the free-boundary compression flow field, the pressure curves in the $\mathrm{Y}$ direction at $2.0 \mathrm{~mm}$ behind the detonation front are obtained (Fig. 9) and normalised by the CJ pressure. As shown by the blue solid line in Fig. 9 at $\mathrm{t}=675 \mu \mathrm{s}$, a high pressure region (with a peak pressure of $2.36 \mathrm{P}_{\mathrm{CJ}}$ ) is formed in the lower half of the flow field behind the detonation front before the detonation wave moves forward. Moreover, the detonation front trajectory corresponds to the position of line (1) in Fig. 8(b). After the detonation front completes a fluctuation, at $\mathrm{t}=715 \mu \mathrm{s}$, the pressure in the lower half of the flow field given by the blue dotted line in Fig. 9 returns to a relatively low state of approximately $1.74 \mathrm{P}_{\mathrm{CJ}}$, and its detonation front trajectory corresponds to the position of line (4) in Fig. 8(b). Similarly, the pressure values at the beginning (740 $\mu \mathrm{s})$ and at 
the end $(740 \mu \mathrm{s})$ of the next fluctuation also present the same trend as the previous one. Their detonation front trajectories correspond to the position of line (5) and (6) in Fig. 8(b), which are similar in shape to line (1) and line (4). In addition, the wave fluctuation period between the blue solid line and the red one in Fig. 9 is approximately $65 \mu \mathrm{s}$, which is equal to the estimated oscillation period (Fig. 6).

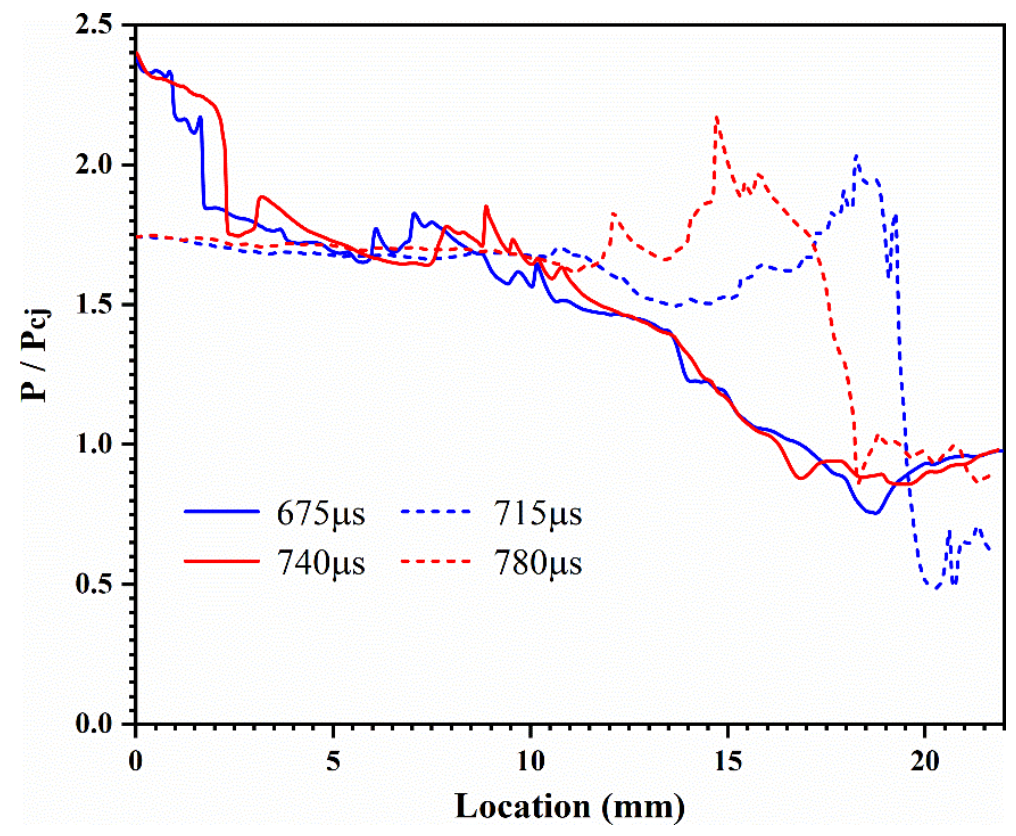

Fig. 9 Pressure curves in Y direction approximately $2.5 \mathrm{~mm}$ behind detonation front of Case 1 .

The consumption of unburned jet is closely related to the turbulent mixing and diffusion effect. To intuitively analyse the consumption rate of unburned jets, the mixing rate is employed, i.e., the reactant mass diffusion flux, representing the intensity of two species mixing caused by diffusion. ${ }^{55}$ In Ref. [56] and Ref. [57], it is stated that for binary mixtures, diffusion velocities are well approximated by Fick's law, thus the diffusive mass flux for reactant is given by $-\rho D \nabla \cdot Y_{1}$. Taking its magnitude, the mixing rate formula is given by Eq. $(13)^{56}$ : 


$$
\text { Mixing rate }=\left|\rho D \nabla \cdot Y_{1}\right| \text {, }
$$

where $\rho$ is the mixture density, $D$ is the diffusion coefficient, and $Y_{1}$ is the mass fraction of the reaction. It has been reported that a high mixing rate lowers the concentration of unburned pockets until mixing is completed. ${ }^{58}$ As indicated by the mixing rate (Fig. 10(a)) and contour of mass fractions of reactants (Fig. 10(b)) at $\mathrm{t}=$ $435 \mu \mathrm{s}$, it is evident that the higher the vortex concentration, the higher the mixing rate of unburned pockets. However, the vortices on the boundary of the unburned jet induced by the velocity shear layer are numerous, whereas those on the unburned jet generated by the Prandtl-Meyer expansion fan are relatively few.

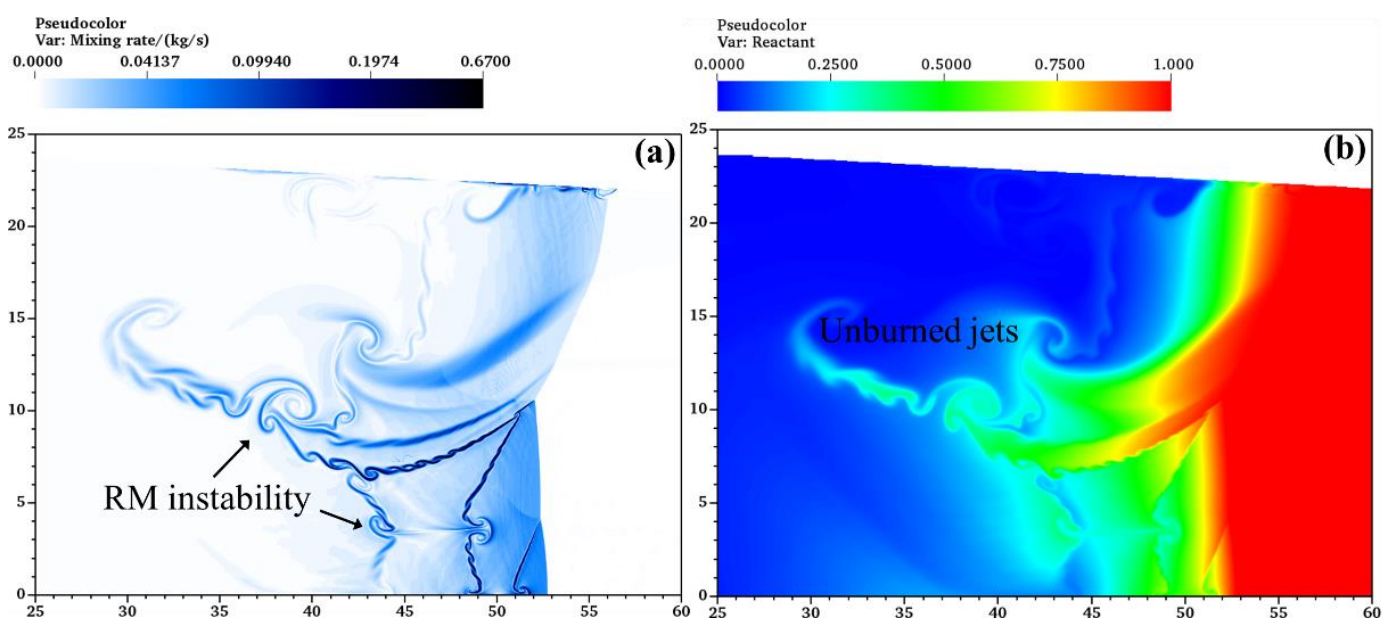

Fig. 10 Mixing rate of reactants (a) and contour of reactant mass fractions (b) at $t=435 \mu$ s of Case 1 .

Fig. 10(a) shows that RM instability vortices exists between the unburned jet and the burned gas. The inconsistency in the directions of density gradient and pressure gradient produces baroclinic vorticity, causing the RM instability at the interface of the unburned jet. ${ }^{59}$ Furthermore, the disturbance on the jet interface continues to induce numerous vortices, thereby promoting the turbulent mixing and diffusion 
effect of these jets. The mechanism of baroclinic effect that induces vorticity can be described by the vortex dynamic equation in fluid mechanics. ${ }^{59}$ In two-dimensional problems, the vorticity caused by stretching and twisting as well as the influence of the initial viscosity terms can be ignored. ${ }^{60}$ The initial vorticity of the interface is not considered. ${ }^{61}$ Thus, the baroclinic vorticity equation is expressed as Eq.(14) ${ }^{59}$ :

$$
\frac{d \vec{\omega}}{d t}_{\text {baroclinic }}=\frac{1}{\rho^{2}} \nabla \rho \times \nabla p,
$$

where $\nabla \rho$ is the density gradient; $\nabla p$ is the pressure gradient, and $\vec{\omega}$ is the vorticity generated at the interface of the unburned jet by the misalignment between the density gradient and pressure gradient. The magnitude of the term in the right hand side of Eq.(14) is $|\nabla \rho| \cdot|\nabla p| \cdot \sin \alpha / \rho^{2}$, where $\alpha$ is the angle between the density gradient and pressure gradient.

Fig. 11 shows the magnitude and factors of the baroclinic vorticity, and the dashed lines in Fig. 11(a) and (b) denote the interface of unburned jets in Fig. 10(b). It is seen from Fig. 11(a) that the baroclinic vorticity exists at the boundaries of both unburned jets. The baroclinic vorticity on the lower boundary of the unburned jet induced by the velocity shear layer is much larger than that on the upper boundary of the unburned jet induced by the expansion fan, as the data shown in Table 5 (data are collected from Fig. 11). Table 5 shows that the baroclinic vorticities at points "I" and "J" are much larger than those at points "G" and "H". The comparison illustrates that the magnitude of the baroclinic vorticity at the interface of the unburned jet is mainly affected by the density gradient and pressure gradient. In fact, the effect of the angle is not decisive as shown in Fig. 11(b) and Table 5. However, it is noted that it can 
directly determine whether the baroclinic vorticity is generated or not, e.g., point "L". These gradients on the lower boundary of the unburned jet are mainly caused by highly unstable shear layers, which are generated by triple points, as depicted in Fig. 11(a) (c) and (d). With the extension of the unburned jet, the effect of the unstable shear layer on the unburned jet is weakened, but the baroclinic vorticity of the point "K" is still greater than that of point "G" and point " $\mathrm{H}$ ". Therefore, the unstable shear layers contribute significantly to the produce of vortices. It can be concluded that the baroclinic mechanism plays a key role in the generation of vortices to accelerate the turbulent mixing of unburned jets. These accelerate the consumption of the unburned pockets and increase the heat release rate behind the detonation front to support the forward propagation of the detonation wave.
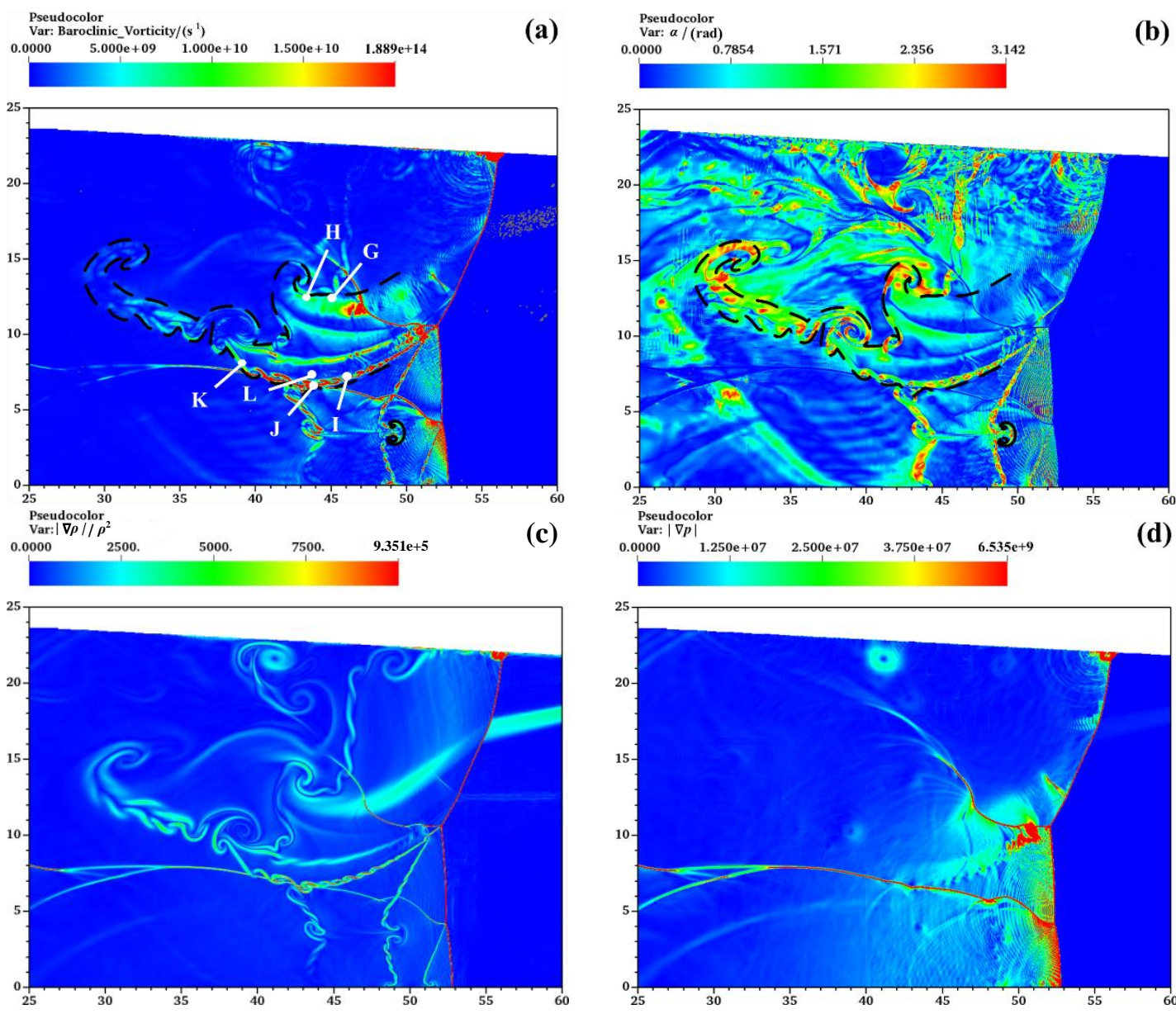

(c)
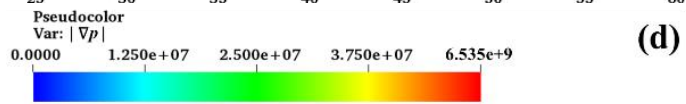

(d)

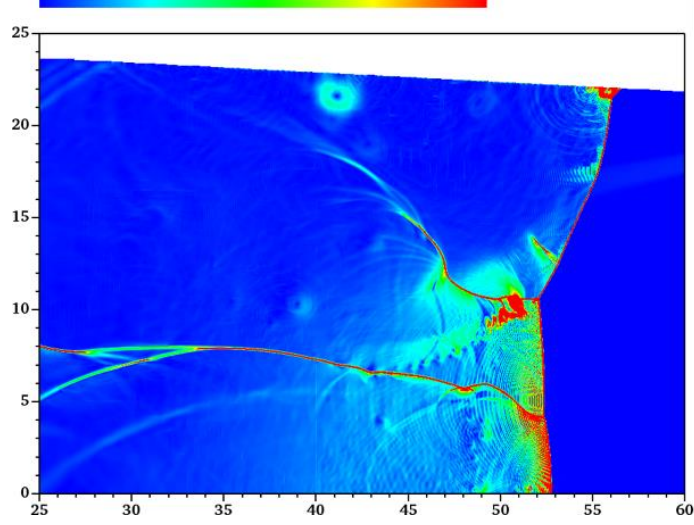


Fig. 11 Contours of magnitude and factors of the baroclinic vorticity at $t=435 \mu$ s of Case 1 , (a) magnitude of the baroclinic vorticity, (b) the angle between density gradient and pressure gradient, (c) the ratio of the magnitude of density gradient to density square, (d) magnitude of pressure gradient.

Table 5 Details of the data points collected from Fig. 11.

\begin{tabular}{cccccc}
\hline Points & $\begin{array}{c}\text { Coordinates } \\
(\mathrm{mm})\end{array}$ & $\begin{array}{c}\text { Baroclinic vorticity } \\
\left(\mathrm{s}^{-1}\right)\end{array}$ & $\alpha(\mathrm{rad})$ & $|\nabla \rho| / \rho^{2}$ & $|\nabla p|$ \\
\hline $\mathrm{G}$ & $(45.1,12.4)$ & $6.09 \times 10^{9}$ & 0.70 & $1.77 \times 10^{3}$ & $5.35 \times 10^{6}$ \\
$\mathrm{H}$ & $(43.3,12.4)$ & $7.79 \times 10^{9}$ & 1.19 & $2.37 \times 10^{3}$ & $3.54 \times 10^{6}$ \\
$\mathrm{I}$ & $(46.1,7.1)$ & $1.93 \times 10^{11}$ & 1.66 & $1.07 \times 10^{4}$ & $1.81 \times 10^{7}$ \\
$\mathrm{~J}$ & $(43.8,6.7)$ & $8.14 \times 10^{10}$ & 2.23 & $9.21 \times 10^{3}$ & $1.12 \times 10^{7}$ \\
$\mathrm{~K}$ & $(39.1,8.0)$ & $1.38 \times 10^{10}$ & 1.15 & $3.83 \times 10^{3}$ & $3.95 \times 10^{6}$ \\
$\mathrm{~L}$ & $(43.6,7.4)$ & 0 & 0 & $6.70 \times 10^{2}$ & $8.61 \times 10^{6}$ \\
\hline
\end{tabular}

Based on the foregoing analyses, the addition of a velocity shear layer can cause the gradually attenuated detonation wave to self-sustain its forward propagation, which cannot be separated from the generation and consumption of the unburned jets. The compression effect of the unburned jet on the flow field, suppresses the expansion of the product behind the detonation front, resulting in increasing the pressure of the flow field. This increased pressure further pushes the detonation wave forward. Furthermore, the misalignment of the interfacial density gradient and pressure gradient induces the baroclinic vorticity, leading to the RM instability on the boundary of unburned jets. Consequently, numerous vortices are induced, thereby enhancing the continuous turbulent mixing effects. The combined effects eventually lead to the self-sustaining propagation in supersonic expanding channels. 


\subsection{Effects of velocity shear layer}

To study the influence of different velocity shear layers on the detonation propagation characteristics, a comparison in which other working conditions (Case 18 in Table 3) are considered is performed. As shown in Fig. 12, the propagation characteristics of the detonation wave in Case 2 exhibit a dynamic process opposite that of Case 1 (Fig. 5). Because of the velocity exchange between the upper and lower halves of the flow field, an asynchronous dynamic propagation process in which the detonation front in the lower half of the flow field first tilts forward and the upper half catches up with that in the lower half of the flow field is observed.

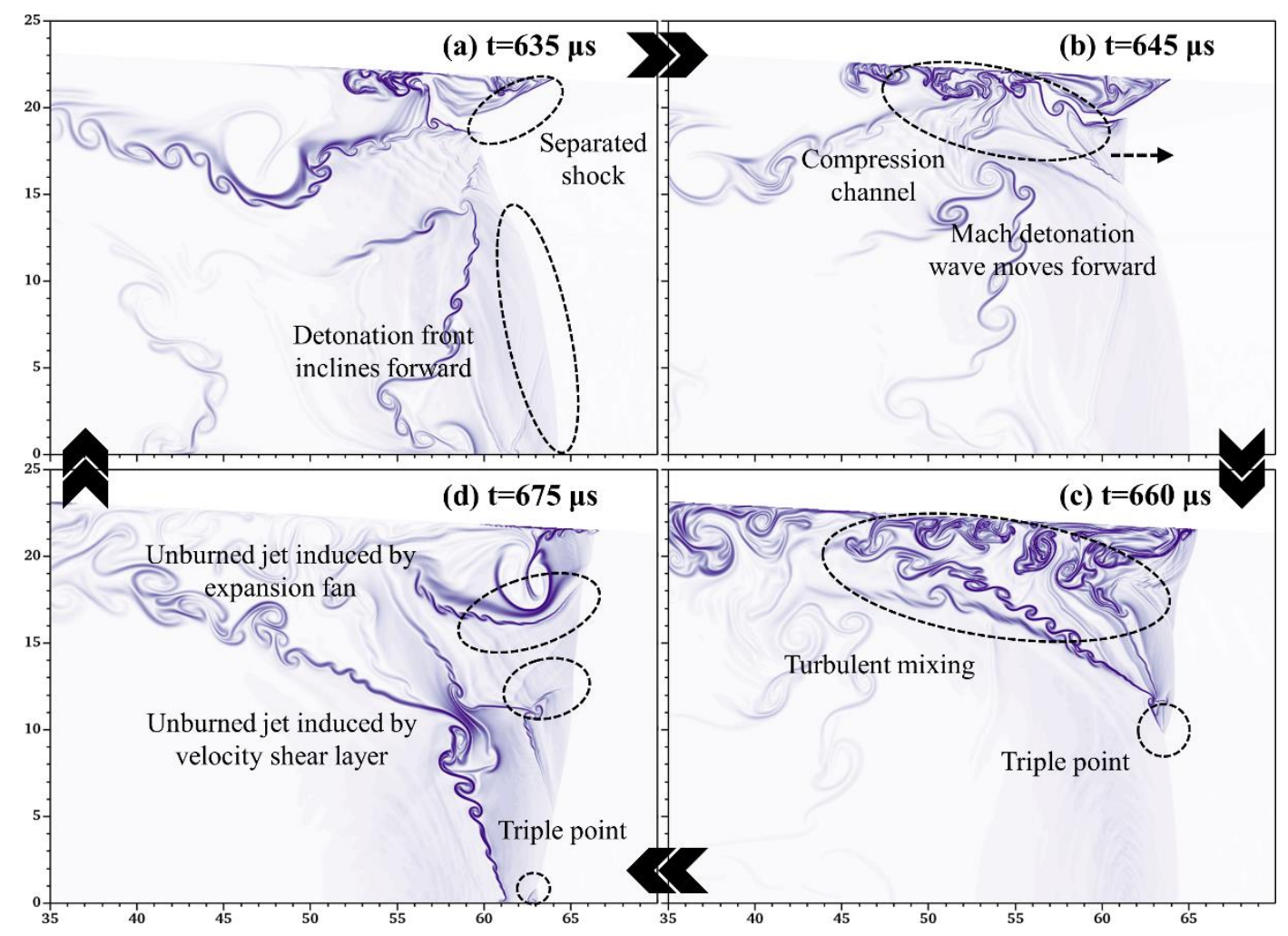

Fig. 12 Numerical species schlieren in Case 2, (a) $t=635 \mu \mathrm{s}$, (b) $\mathrm{t}=645 \mu \mathrm{s}$, (c) $\mathrm{t}=660 \mu \mathrm{s}$, (d) $\mathrm{t}=$ $675 \mu \mathrm{s}$.

The trajectories of the detonation front influenced by different velocity shear 
layers are illustrated in Fig. 13. The comparison suggests that the propagation of the detonation front is affected by the average flow field velocity (V) and the spatial distribution of velocity. When the average velocity $(\mathrm{V})$ of the flow field is less than the CJ velocity (i.e., Case 1 and Case 2), the self-sustained detonation wave propagates forward at a relatively constant speed, where the relative velocity of Case 2 is $59.25 \mathrm{~m} / \mathrm{s}$. Under the condition of $\mathrm{V}=\mathrm{V}_{\mathrm{CJ}}$, the detonation waves in Case 3 and Case 4 are relatively stable at approximately $X=46.0 \mathrm{~mm}$ and $X=50.6 \mathrm{~mm}$, respectively. This suggests the formation of a dynamically stationary detonation in the expanding channel. This dynamically stationary detonation lasts longer than the uniform case shown in Fig. 6. When V exceeds the CJ velocity (i.e., Case 5-Case 8), the detonation wave tends to attenuate with an attenuation rate which is proportional to the average velocity. Among these cases, the detonation waves in Case 7 and Case 8 attenuate at approximately $\mathrm{X}=4.5 \mathrm{~mm}$; thereafter, the backward propagation is converted to forward propagation. If the simulation time of Case 5 and Case 6 is extended, a self-sustained forward propagation can also be observed. However, the reliability of the results is questionable, which may be caused by the one-step reaction model. Because of the continuous reaction profile of the one-step model, the detonation cannot be quenched if the growth time is long enough. ${ }^{30}$ In the next step, a detailed chemical reaction kinetic model will be adopted to simulate the detonation in non-uniform supersonic flow. The fluctuation of the detonation front caused by velocity layers may be reasonably assumed as reducing the pressure oscillations caused by the rapid combustion of unburned pockets to promote the propagation of 
the detonation wave.

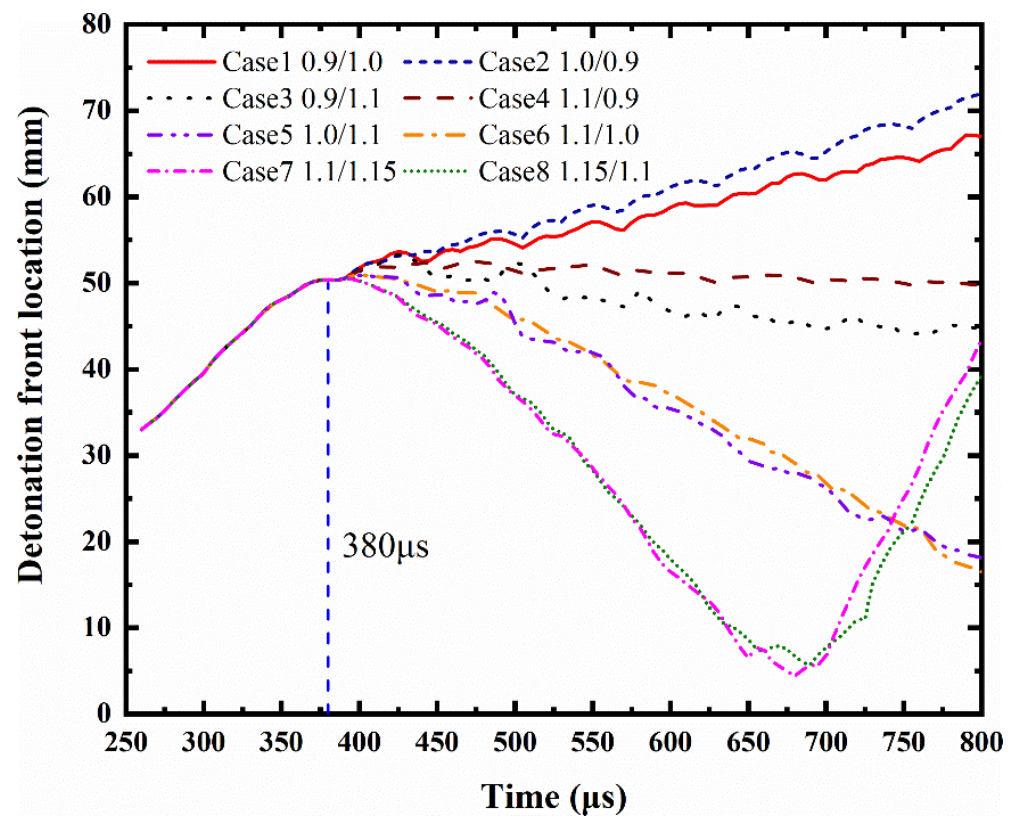

Fig. 13 Trajectories of detonation front under different velocity shear layers (the ratio in the legend is

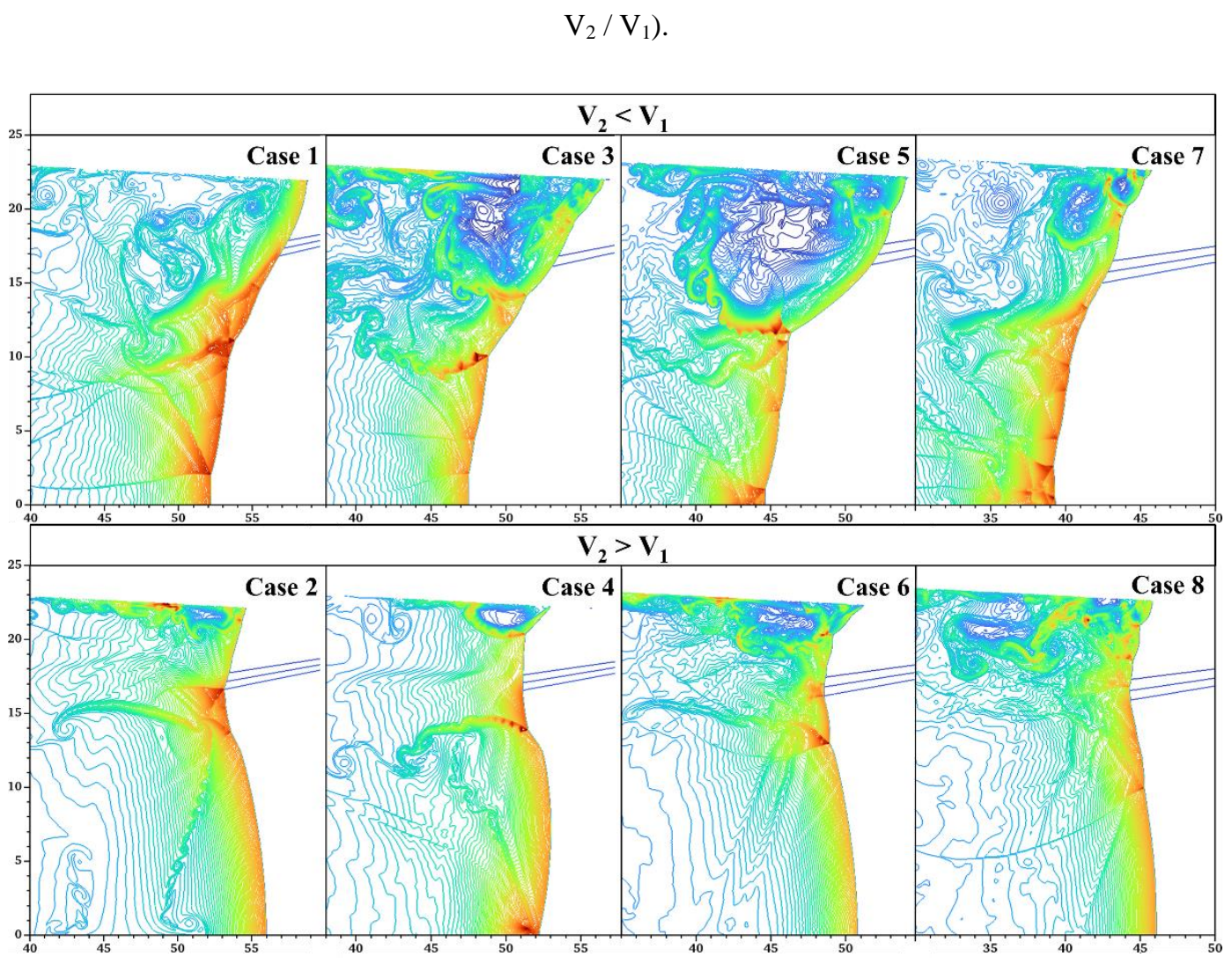

Fig. 14 Density isolines of typical detonation wave structures in Case 1-Case 8.

The typical detonation wave structures of Case 1-Case 8 shown in Fig. 14 
clearly indicate that the different velocity shear layers result in the variable structures of the detonation front. Consider the case $\mathrm{V}_{2}<\mathrm{V}_{1}$ as an example. The phenomenon illustrated in Fig. 14 is caused by the lower total pressure in the upper half of the flow field than that in the lower half. According to Eq.(12), to achieve a pressure matching behind the shock front, the detonation wave propagates forward in the upper half of the flow field to increase the relative Mach number. This improves the strength of the detonation wave, and finally achieves the pressure matching of the wave in the upper and lower flow fields. The opposite is true when $V_{2}>V_{1}$. Eventually, the detonation wave dynamically propagates in a whole. Moreover, the larger the difference between $\mathrm{V}_{1}$ and $\mathrm{V}_{2}$, the more unstable the detonation front, and the greater the fluctuation.

\section{Conclusion}

In the present work, the dynamic adaptive mesh refinement method along with the two-dimensional Navier-Stokes equations are solved to investigate the detonation propagation in supersonic flow with velocity shear layer in an expanding combustor. The mechanism of self-sustaining propagation is examined and the influence of different velocity shear layers is analysed. The main conclusions derived are as follows:

1) There are two different unburned jets behind the detonation front in a supersonic expanding combustor with velocity shear layer. These jet structures are induced by the velocity shear layer and Prandtl-Meyer expansion fan. The two jets interact and mix gradually. The detonation presents an asynchronous dynamic propagation mode, which reduces the attenuation effect caused by the rapid 
consumption of unburned jets, further promoting the self-sustaining propagation of the detonation wave.

2) Because of the further reduction of the space behind the detonation front that is compressed by the free boundary of the unburned jet, a micro-compression channel is formed between the free boundary and channel wall. This increases the pressure behind the detonation front by suppressing the expansion of products, thereby promoting the forward propagation of detonation.

3) The RM instability induced by the baroclinic mechanism on the boundary of the unburned jets generates numerous vortices, which cause considerable turbulent mixing between burned and unburned gases. This contributes to the consumption of unburned jets, which intensifies heat release, thereby supports the self-sustaining propagation of the detonation wave.

4) The detonation front exhibits different propagation characteristics under the influence of various velocity shear layers. To achieve the pressure matching behind the detonation front, the detonation front in the upper half of the flow field tilts forward when $\mathrm{V}_{2}<\mathrm{V}_{1}$; the opposite is true when $\mathrm{V}_{2}>\mathrm{V}_{1}$. In addition, the larger the difference among the velocity shear layers, the greater the fluctuation of the detonation front.

\section{Acknowledgment}

This work is supported by National Natural Science Foundation of China (No. $11702323)$. 


\section{Conflict of interest}

The authors have no conflicts to disclose.

\section{Data availability}

The data used to support the findings of this study are available from the corresponding author upon request.

\section{References}

${ }^{1}$ Q. Liu, D. Baccarella, T. Lee, "Review of combustion stabilization for hypersonic airbreathing propulsion,” Prog. Aerosp. Sci. 119, 100636 (2020).

${ }^{2}$ K. Kailasanath, "Review of Propulsion Applications of Detonation Waves," AIAA J. 38, 1698-1708 (2000).

${ }^{3}$ E. T. Curran, W. H. Heiser, and D. T. Pratt, "Fluid phenomena in scramjet combustion systems," Annu. Rev. Fluid Mech. 28, 323-360 (1996).

${ }^{4} \mathrm{~K}$. Kailasanath, "Recent developments in the research on pulse detonation engines," AIAA J. 41, 145159 (2003).

${ }^{5} \mathrm{~K}$. Wang, Z. Zhang, P. Yang, and H. Teng, "Numerical study on reflection of an oblique detonation wave on an outward turning wall," Phys. Fluids 32, 46101 (2020).

${ }^{6} \mathrm{Y}$. Fang, Y. Zhang, X. Deng, and H. Teng, "Structure of wedge-induced oblique detonation in acetylene-oxygen-argon mixtures," Phys. Fluids 31, 26108 (2019).

${ }^{7}$ Y. Liu, W. Zhou, Y. Yang, Z. Liu, and J. Wang, "Numerical study on the instabilities in $\mathrm{H}_{2}$-air rotating detonation engines," Phys. Fluids 30, 46106 (2018).

${ }^{8}$ T. H. Yi, F. K. Lu, D. R. Wilson, and G. Emanuel, "Numerical study of detonation wave propagation in a confined supersonic flow," Shock Waves 27, 395-408 (2017).

${ }^{9} \mathrm{G}$. B. Goodwin and E. S. Oran, "Premixed flame stability and transition to detonation in a supersonic combustor,” Combust. Flame 197, 145-160 (2018).

${ }^{10}$ D. Cuppoletti, T. Ombrello, C. Carter, S. Hammack, and J. Lefkowitz, "Ignition dynamics of a pulse detonation igniter in a supersonic cavity flameholder," Combust. Flame 215, 376-388 (2020).

${ }^{11} \mathrm{M}$. Kellenberger and G. Ciccarelli, "Three-dimensional behaviour of quasi-detonations," Combust. Flame 215, 145-156 (2020).

${ }^{12}$ W. Chen, J. Liang, X. Cai, and Z. Lin, "Detonation simulations in expanding channel with supersonic combustible mixture,” Int. J. Hydrogen Energy 42, 6384-6393 (2017).

${ }^{13} \mathrm{Q}$. Wang, Z. Wang, and Y. Zhao, "The impact of streamwise convex curvature on the supersonic turbulent boundary layer," Phys. Fluids 29, 116106 (2017).

${ }^{14} \mathrm{X}$. Wang, Z. Wang, M. Sun, Q. Wang, and Z. Hu, "Effects of favorable pressure gradient on turbulence structures and statistics of a flat-plate supersonic turbulent boundary layer," Phys. Fluids 32 , 
25107 (2020).

${ }^{15} \mathrm{~V}$. A. Subbotin, "Triggering of Detonation upon Flame Interaction with an Expansion Wave," Combust. Explos. Shock Waves 39, 91-101 (2003).

${ }^{16}$ Y. V. Tunil, G. Y. Gerasimov, V. Y. Levashov, and N. A. Slavinskaya, "Numerical Simulation of Detonation Combustion of Kerosene Vapors in an Expanding Nozzle," Combust. Explos. Shock Waves 56, 344-352 (2020).

${ }^{17} \mathrm{Yu}$. V. Tunik, "Detonation combustion of hydrogen in a convergent-divergent nozzle with a central coaxial cylinder," Fluid Dyn. 49, 688-693 (2014).

${ }^{18} \mathrm{~K}$. Wang, H. Teng, P. Yang, and H. D. Ng, "Numerical investigation of flow structures resulting from the interaction between an oblique detonation wave and an upper expansion corner," J. Fluid Mech. 903, A28 (2020)

${ }^{19} \mathrm{X}$. Cai, R. Deiterding, J. Liang, D. Dong, and M. Sun, "Dynamic detonation stabilization in supersonic expanding channels," Phys. Rev. Fluids 4, 083201 (2019).

${ }^{20} \mathrm{C}$. M. Brophy and R. K. Hanson, "Fuel distribution effects on pulse detonation engine operation and performance,” J. Propul. Power 22, 1155-1161 (2006).

${ }^{21}$ B. A. Rankin, D. R. Richardson, A. W. Caswell, A. G. Naples, J. L. Hoke, and F. R. Schauer, "Chemiluminescence imaging of an optically accessible non-premixed rotating detonation engine," Combust. Flame 176, 12-22 (2017).

${ }^{22} \mathrm{C}$. Wang, J. Li, Z. Tang, Y. Zhuang, and J. Guo, "Flame propagation in methane-air mixtures with transverse concentration gradients in horizontal duct," Fuel 265, 116926 (2020).

${ }^{23} \mathrm{~K}$. Kumaran and V. Babu, "Mixing and combustion characteristics of kerosene in a model supersonic combustor,” J. Propul. Power 25, 583-592 (2009).

${ }^{24}$ Y. Wang, W. Han, R. Deiterding, and Z. Chen, "Effects of disturbance on detonation initiation in $\mathrm{H}_{2} / \mathrm{O}_{2} / \mathrm{N}_{2}$ mixture," Phys. Rev. Fluids 3, 123201 (2018).

${ }^{25} \mathrm{M}$. Sun, Z. Hu, and N. D. Sandham, "Recovery of a supersonic turbulent boundary layer after an expansion corner," Phys. Fluids 29, 76103 (2017).

${ }^{26} \mathrm{M}$. Sun, Y. Liu, and Z. Hu, "Turbulence decay in a supersonic boundary layer subjected to a transverse sonic jet," J. Fluid Mech. 867, 216-249 (2019).

${ }^{27}$ T. A. Khmel and S. I. Tolkacheva, "Model of reduced kinetics for describing heterogeneous detonation in gas particle mixtures with non-uniform concentration distribution," J. Phys. Conf. Ser. 1382, 12102 (2019).

${ }^{28} \mathrm{C}$. Qi, P. Dai, H. Yu, and Z. Chen, "Different modes of reaction front propagation in n-heptane/air mixture with concentration non-uniformity," Proc. Combust. Inst. 36, 3633-3641 (2017).

${ }^{29} \mathrm{Z}$. Yu, H. Zhang and P. Dai, "Autoignition and detonation development induced by temperature gradient in $\mathrm{n}-\mathrm{C}_{7} \mathrm{H}_{16} /$ air/ $\mathrm{H}_{2} \mathrm{O}$ mixtures," Phys. Fluids, 33, 017111 (2021).

${ }^{30} \mathrm{Q}$. Song, Y. Han, and W. Cao, "Numerical investigation of self-sustaining modes of 2D planar detonations under concentration gradients in hydrogen-oxygen mixtures," Int. J. Hydrogen Energy 45, 29606-29615 (2020).

${ }^{31}$ M. S. Kuznetsov, V. I. Alekseev, S. B. Dorofeev, I. D. Matsukov, and J. L. Boccio, "Detonation propagation, decay, and reinitiation in nonuniform gaseous mixtures," Symp. (Int.) Combust. 27, 22412247 (1998).

${ }^{32}$ Y. M. Kim, S. J. Kim, Z. J. Chen, and C. P. Chen, "Numerical simulation of combustion wave propagation in an air-fuel spray mixture with temperature nonuniformity," Numer. Heat Tr. A-Appl. 34, 23-41 (1998). 
${ }^{33}$ X. Cai, J. Liang, R. Deiterding, and Z. Lin, "Detonation simulations in supersonic combustible mixtures with nonuniform species," AIAA J. 54, 2449-2462 (2016).

${ }^{34}$ X. Cai, J. Liang, Z. Lin, R. Deiterding, and F. Zhuang, "Detonation initiation and propagation in nonuniform supersonic combustible mixtures," Combust. Sci. and Technol. 187, 525-536 (2015).

${ }^{35}$ Y Huang, X He, Y Jin, H Zhu, and Z Zhu. "Effect of non-uniform inlet profile on the combustion performance of an afterburner with bluff body," Energy 216, 119142 (2021).

${ }^{36} \mathrm{~S}$ Kadowaki and H Kobayashi, "Dynamic behavior of premixed flames propagating in non-uniform velocity fields — assessment of intrinsic instability in turbulent combustion_-, Trans. Japan Soc. Aero. Space Sci. 51, 244-251 (2009).

${ }^{37}$ X. Cai, R. Deiterding, J. Liang, M. Sun, and Y. Mahmoudi, "Diffusion and mixing effects in hot jet initiation and propagation of hydrogen detonations,” J. Fluid Mech. 836, 324 (2018).

${ }^{38}$ R. Deiterding, M. O. Domingues, and K. Schneider, "Multiresolution analysis as a criterion for effective dynamic mesh adaptation-A case study for Euler equations in the SAMR framework AMROC," Comput. Fluids 205, 104583 (2020).

${ }^{39}$ X. Cai, R. Deiterding, J. Liang, M Sun, and D Dong, "Detonation stabilization in supersonic flow: effects of suction boundaries," AIAA J. 58, 1348-1355 (2020).

${ }^{40} \mathrm{R}$. Deiterding, "A parallel adaptive method for simulating shock-induced combustion with detailed chemical kinetics in complex domains," Comput. Struct. 87, 769-783 (2009).

${ }^{41}$ J. L. Ziegler, R. Deiterding, J. E. Shepherd, and D.I. Pullin, “An adaptive high-order hybrid scheme for compressive, viscous flows with detailed chemistry," J. Comput. Phys. 230, 7598-7630 (2011).

${ }^{42}$ X. Cai, J. Liang, R. Deiterding, Y. Mahmoudi, and M. Sun, "Experimental and numerical investigations on propagating modes of detonations: Detonation wave/boundary layer interactions," Combust. Flame 190, 201-215 (2018).

${ }^{43}$ J. Liang, X. Cai, Z. Lin, and R. Deiterding, "Effects of a hot jet on detonation initiation and propagation in supersonic combustible mixtures," Acta Astronaut. 105, 265 (2014).

${ }^{44}$ Y. Zhang, L. Zhou, J. Gong, H. D. Ng, and H. Teng, "Effects of activation energy on the instability of oblique detonation surfaces with a one-step chemistry model," Phys. Fluids 30, 106110 (2018).

${ }^{45}$ W. Chen, J. Liang, X. Cai, and Y. Mahmoudi, "Three-dimensional simulations of detonation propagation in circular tubes: Effects of jet initiation and wall reflection,” Phys. Fluids 32, 46104 (2020).

${ }^{46} \mathrm{C}$. Chiquete and M. Short, "Characteristic path analysis of confinement influence on steady twodimensional detonation propagation,” J. Fluid Mech. 863, 789-816 (2019).

${ }^{47}$ D. J. Hill and D. I. Pullin, "Hybrid tuned center-differencer-WENO method for large eddy simulations in the presence of strong shocks," J. Comput. Phys. 194, 435-450 (2004).

${ }^{48}$ C. Pantano, R. Deiterding, D. J. Hill and D.I. Pullin, “A low numerical dissipation patch-based adaptive mesh refinement method for large-eddy simulation of compressible flows," J. Comput. Phys. 221, 63-87 (2007).

${ }^{49}$ X. Cai, R. Deiterding, J. Liang, and Y. Mahmoudi, “Adaptive simulations of viscous detonations initiated by a hot jet using a high-order hybrid WENO-CD scheme," Proc. Combust. Inst. 36, 2725 (2017).

${ }^{50}$ M. V. Silnikov and M. V. Chernyshov, "The interaction of a Prandtl-Meyer wave and a quasi-onedimensional flow region," Acta Astronaut. 109, 248-253 (2015).

${ }^{51}$ P. E. Dimotakis, “Turbulent mixing,” Annu. Rev. Fluid Mech. 37, 329-356 (2005).

${ }^{52}$ E. S. Oran, Jr. J. W. Weber, E. I. Stefaniw, M. H. Lefebvre, and Jr. J. D. Anderson, “A numerical 
study of a two-dimensional $\mathrm{H}_{2}-\mathrm{O}_{2}$-Ar detonation using a detailed chemical reaction model," Combust. Flame 113, 147-163 (1998).

${ }^{53}$ N. D. Domel, "General three-dimensional relation for oblique shocks on swept ramps," AIAA J. 54, 310-319 (2016).

${ }^{54} Y$ Mahmoudi and K. Mazaheri, "High resolution numerical simulation of the structure of 2-D gaseous detonations," Proc. Combust. Inst., 33, 2187-2194 (2011).

${ }^{55}$ C. Tomkins, S. Kumar, G. Orlicz, and K. Prestridge, "An experimental investigation of mixing mechanisms in shock-accelerated flow," J. Fluid Mech. 611, 131-150 (2008).

${ }^{56} \mathrm{M}$. Groom and B. Thornber, "Direct numerical simulation of the multimode narrowband RichtmyerMeshkov instability," Comput. Fluids 194, 104309 (2019).

${ }^{57}$ B. J. Olson and J. Greenough, "Large eddy simulation requirements for the Richtmyer-Meshkov instability," Phys. Fluids 26, 44103 (2014).

${ }^{58}$ M. Stöhr, C. M. Arndt, and W. Meier, "Transient effects of fuel-air mixing in a partially-premixed turbulent swirl flame," Proc. Combust. Inst. 35, 3327-3335 (2015).

${ }^{59}$ N. Peng, Y. Yang, J. Wu and Z. Xiao, "Mechanism and modelling of the secondary baroclinic vorticity in the Richtmyer-Meshkov instability,”. J. Fluid Mech. 911, A56 (2021).

${ }^{60}$ L. Zou, M. Al-Marouf, W. Cheng, R. Samtaney, J. Ding, and X. Luo, "Richtmyer-Meshkov instability of an unperturbed interface subjected to a diffracted convergent shock," J. Fluid Mech. 879, 448-467 (2019).

${ }^{61}$ A. M. Rasmus, C. A. Di Stefano, K. A. Flippo, F. W. Doss, J. L. Kline, J. D. Hager, E. C. Merritt, T. R. Desjardins, W. C. Wan, T. Cardenas, D. W. Schmidt, P. M. Donovan, F. Fierro, J. I. Martinez, J. S. Zingale, and C. C. Kuranz, "Shock-driven discrete vortex evolution on a high-Atwood number oblique interface,” Phys. Plasmas 25, 32119 (2018). 


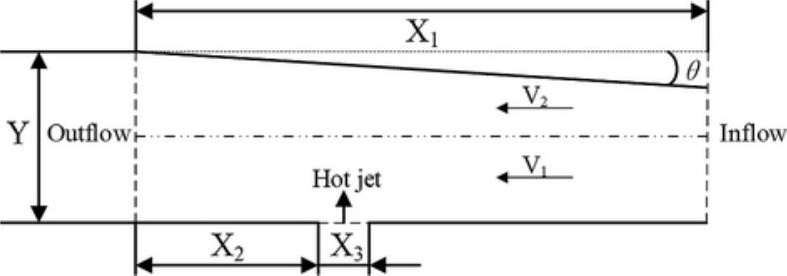




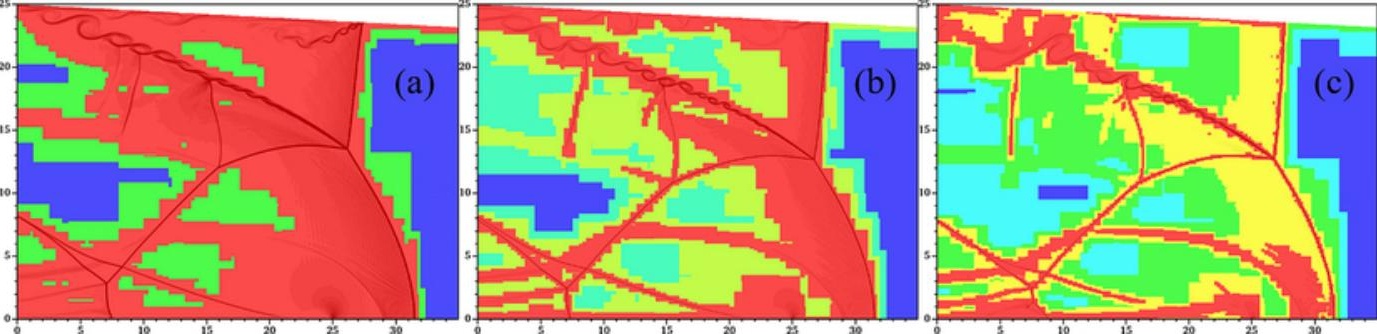




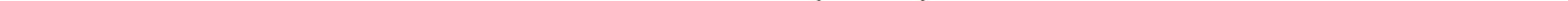




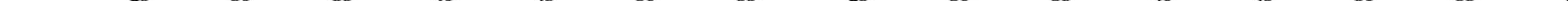




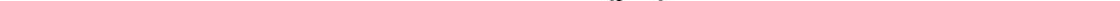


Pseudocolor

Var: Density $/\left(\mathrm{kg} / \mathrm{m}^{3}\right)$

$0.05164 \quad 0.1818$

0.3120

0.4421

0.5723 !

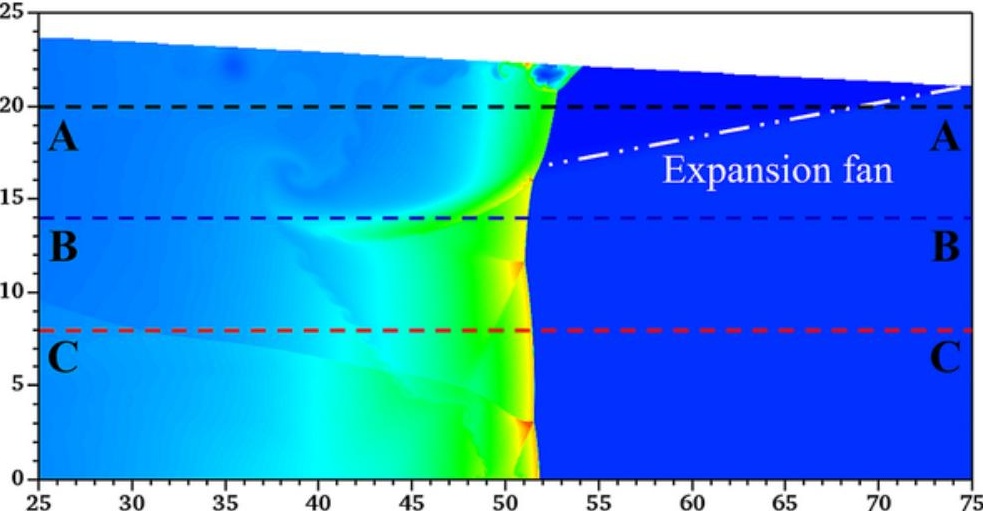


Pseudocolor

Var: Density $/\left(\mathrm{kg} / \mathrm{m}^{3}\right)$

\subsection{8}

0.2179

0.3822

0.5464

0.7107

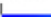

।

।
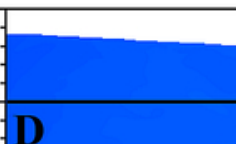

$15-$

E

0

Velocity shear layer 


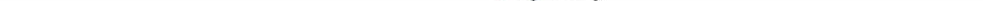




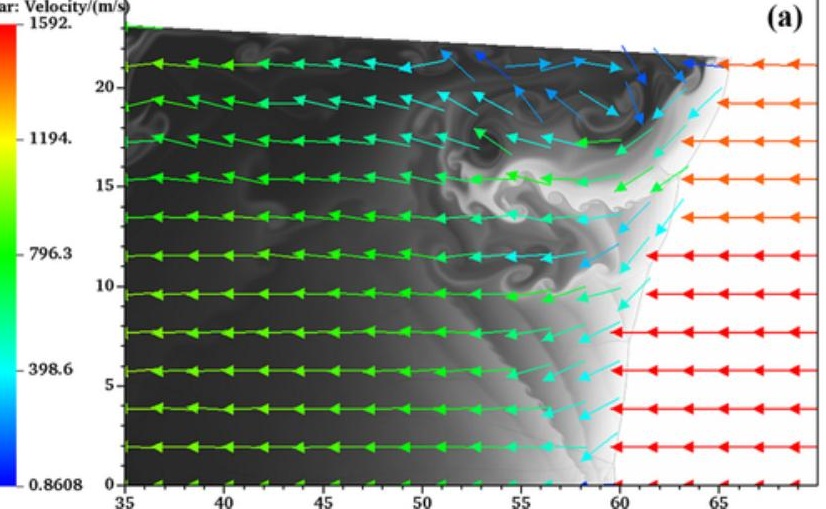

(a)

(b)

(1) $\mathrm{t}=675 \mu \mathrm{s}$ (2) $\mathrm{t}=685 \mu \mathrm{s}$

(3) $\mathrm{t}=700 \mu \mathrm{s}$ (4) $\mathrm{t}=715 \mu \mathrm{s}$

(5) $\mathrm{t}=740 \mu \mathrm{s}$ (6) $\mathrm{t}=780 \mu \mathrm{s}$

$\frac{0+}{35}-40-45-50$

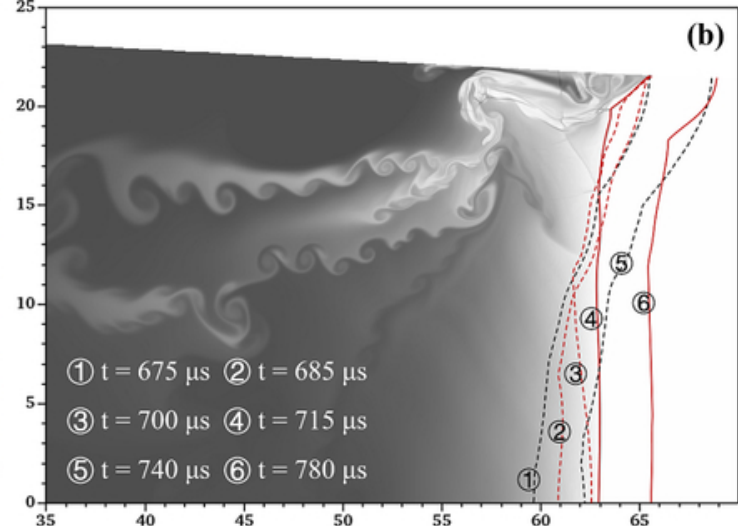




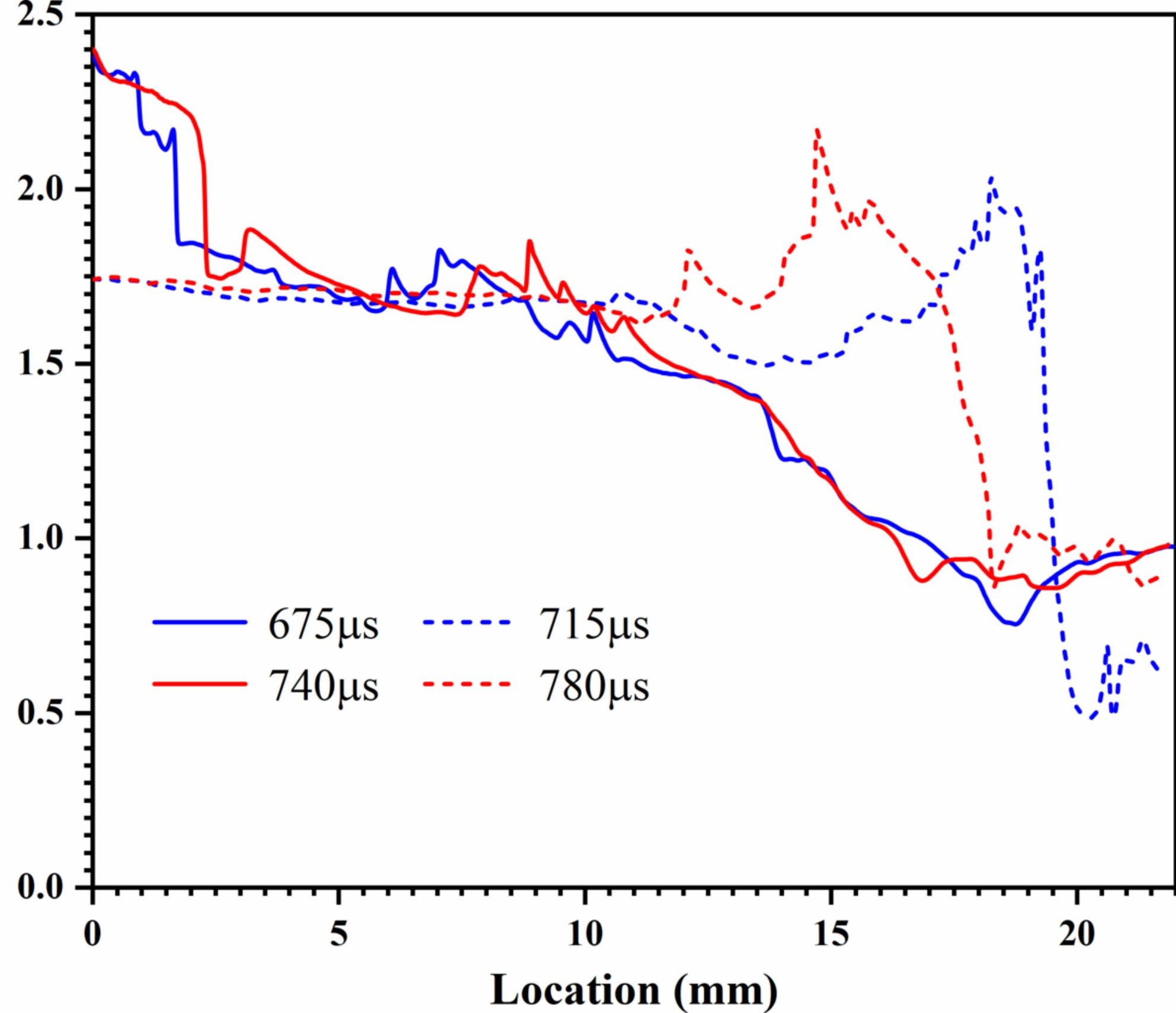




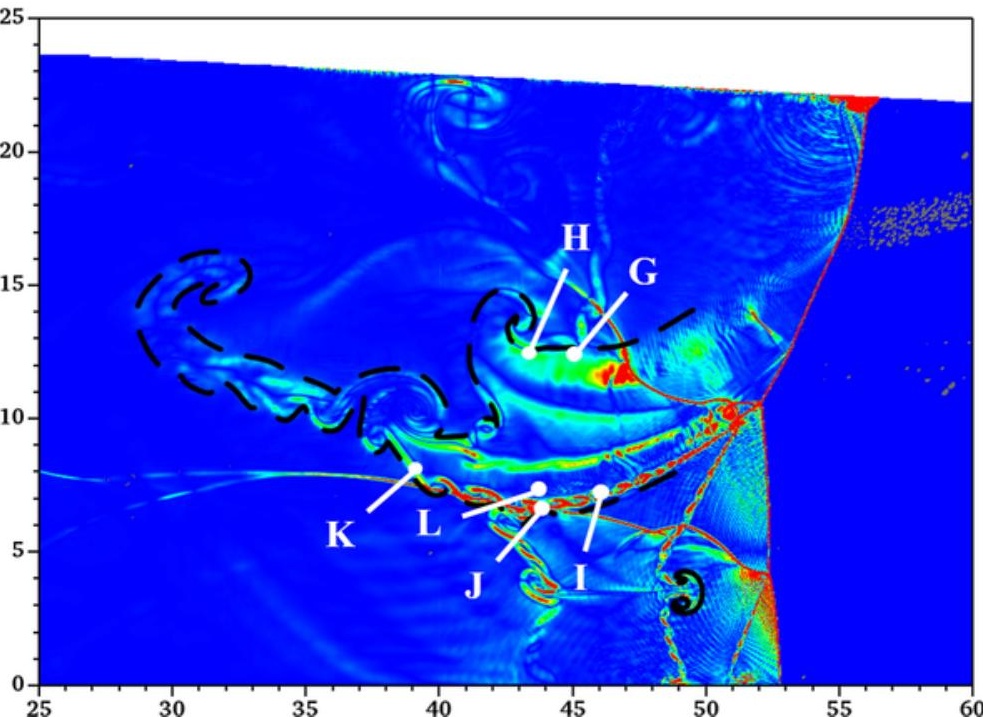




\subsection{0}

0.7854

1.571

2.356

3.142

(b)

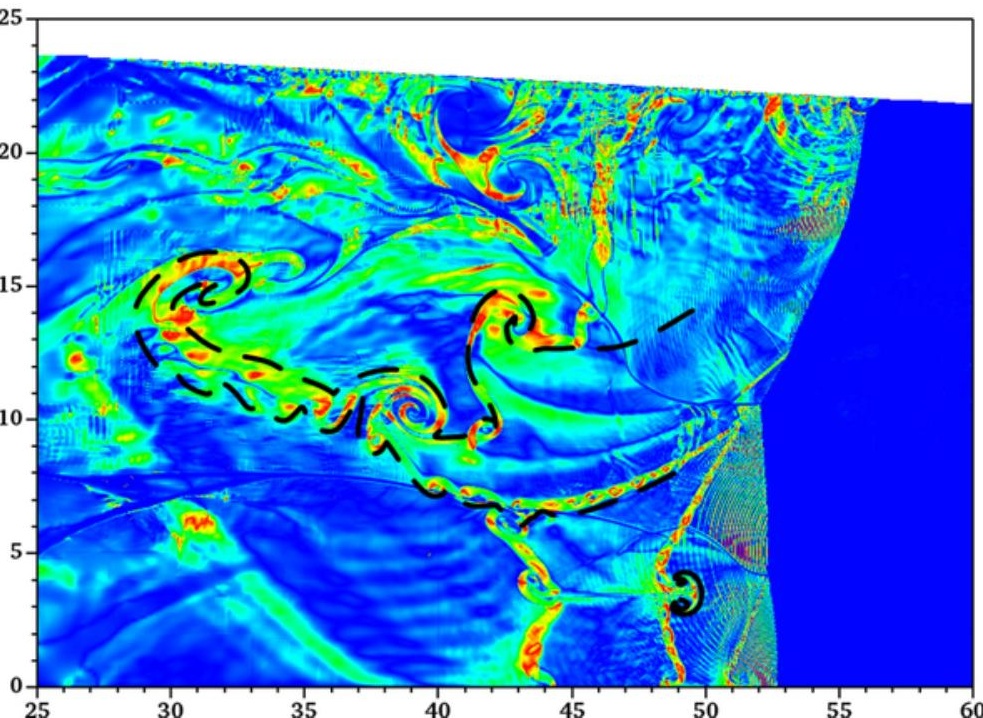


Pseudocolor

Var: $|\nabla \rho| / \rho^{2}$

\subsection{0}

2500.

5000.

7500.

$9.351 \mathrm{e}+5$

(c)

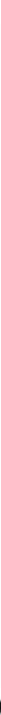


Pseudocolor

Var: $|\nabla p|$

\subsection{0}

$1.250 \mathrm{e}+07$

$2.500 e+07$

$3.750 \mathrm{e}+07$

$6.535 \mathrm{e}+9$

(d)

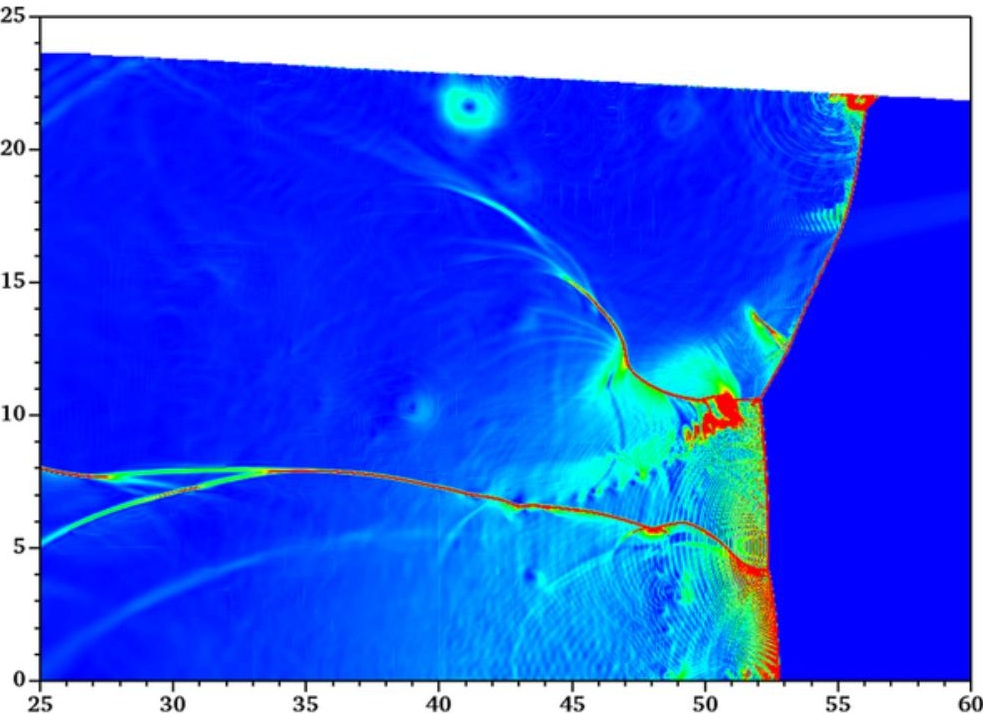




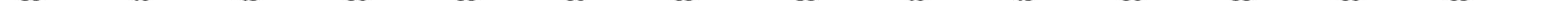




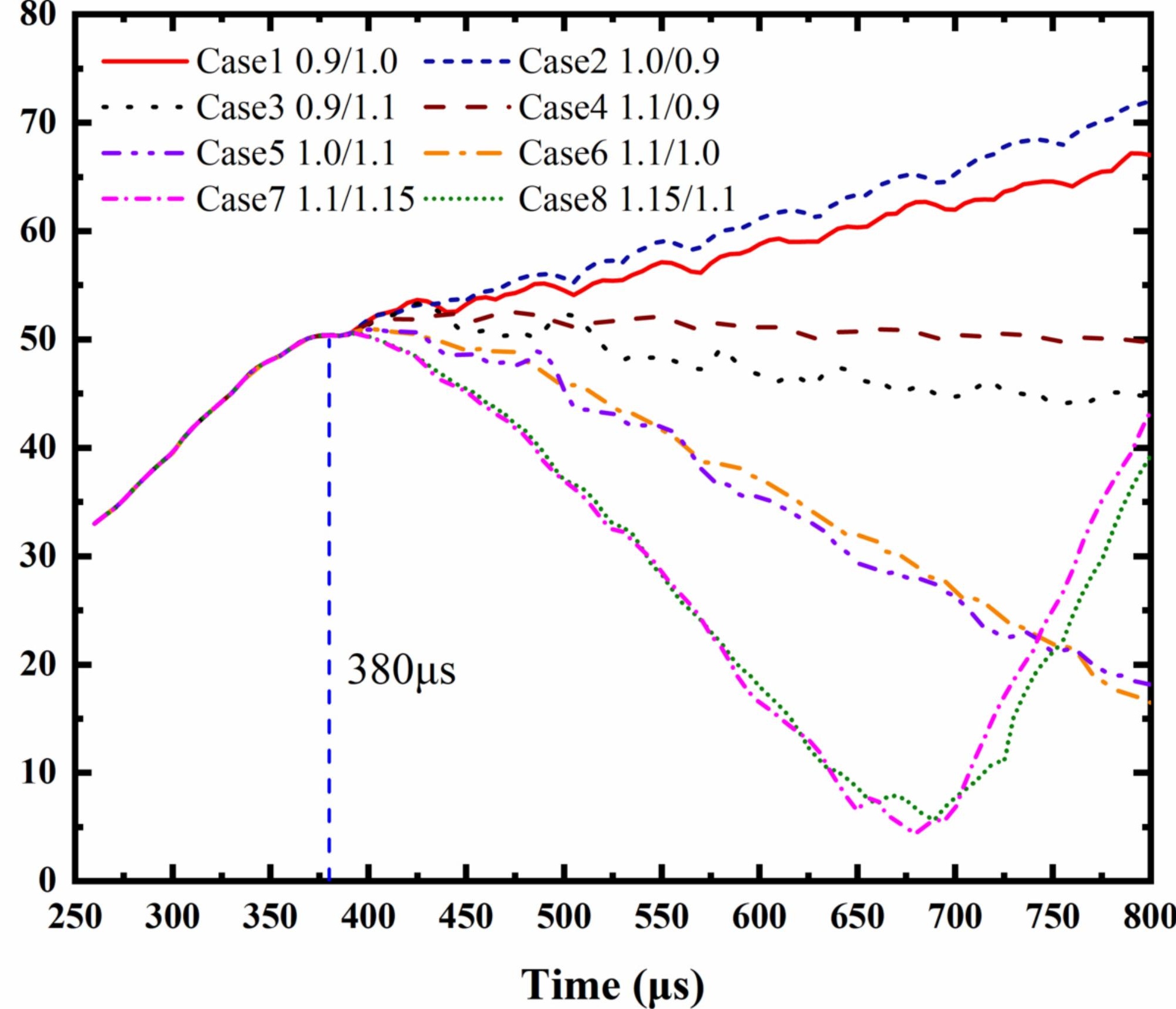




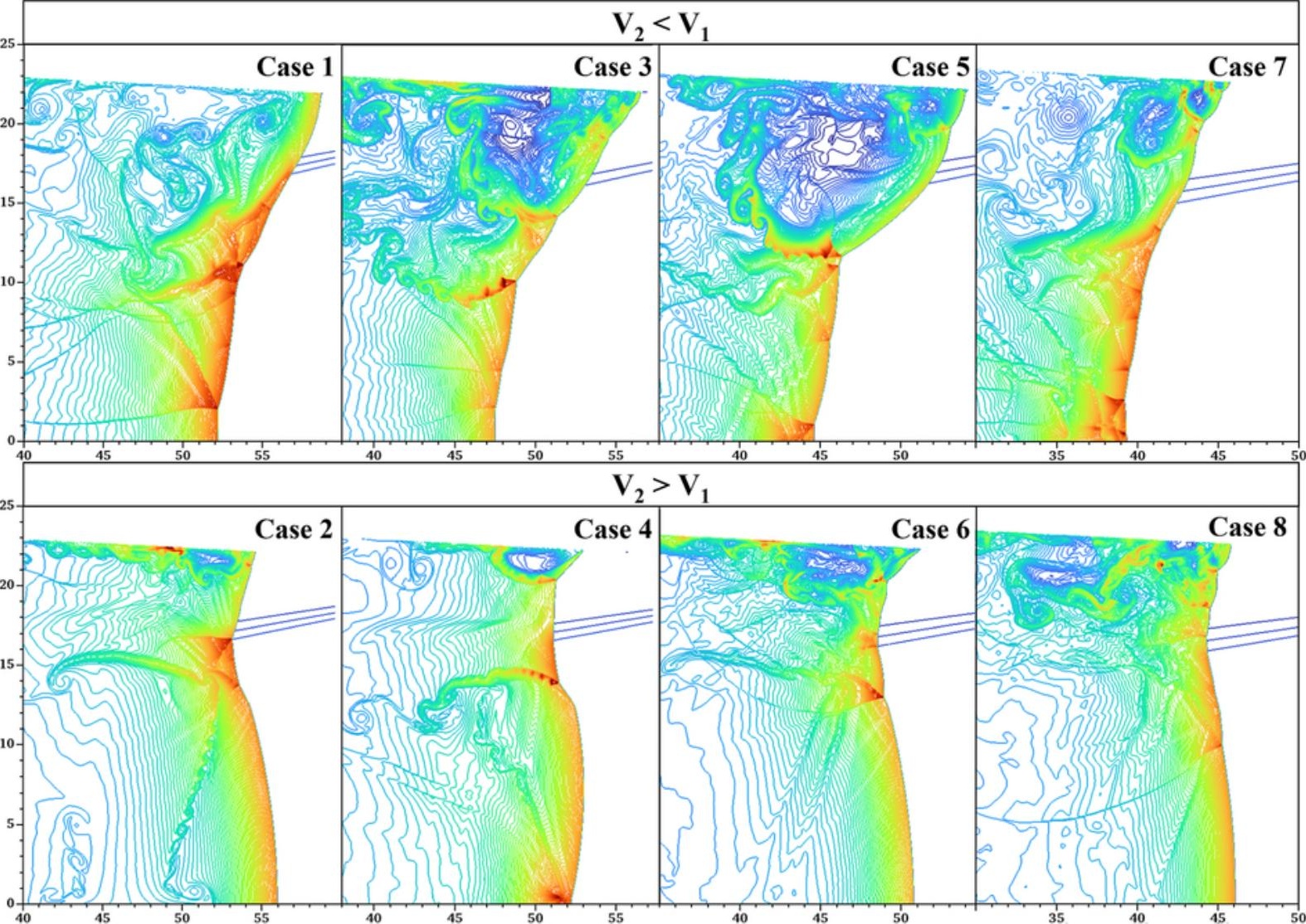

